\title{
STOCHASTIC MODELING OF DENSITY-DEPENDENT DIPLOID POPULATIONS AND THE EXTINCTION VORTEX
}

\author{
CAMILLE CORON, * École Polytechnique
}

\begin{abstract}
We model and study the genetic evolution and conservation of a population of diploid hermaphroditic organisms, evolving continuously in time and subject to resource competition. In the absence of mutations, the population follows a three-type, nonlinear birth-and-death process, in which birth rates are designed to integrate Mendelian reproduction. We are interested in the long-term genetic behavior of the population (adaptive dynamics), and in particular we compute the fixation probability of a slightly nonneutral allele in the absence of mutations, which involves finding the unique subpolynomial solution of a nonlinear three-dimensional recurrence relationship. This equation is simplified to a one-dimensional relationship which is proved to admit exactly one bounded solution. Adding rare mutations and rescaling time, we study the successive mutation fixations in the population, which are given by the jumps of a limiting Markov process on the genotypes space. At this time scale, we prove that the fixation rate of deleterious mutations increases with the number of already fixed mutations, which creates a vicious circle called the extinction vortex.
\end{abstract}

Keywords: Population genetics; diploid population; nonlinear birth-and-death process; fixation probability; Dirichlet problem; multidimensional nonlinear recurrence equation; extinction vortex

2010 Mathematics Subject Classification: Primary 60J10; 60J80

Secondary 60J27; 92D25; 92D15

\section{Introduction}

Our goal is to model a finite population with diploid reproduction and competition. We especially want to understand the role of diploidy and Mendelian reproduction on mutation fixation probabilities and on the genetic evolution of a population. We are interested in studying the progressive accumulation of small deleterious mutations which generates an extinction vortex in small populations (see [7], [13], and [19] for more biological context and analyses).

The population follows a birth-and-death process in which each individual has a natural death rate that depends on its genotype (Section 2). Birth rates are designed to model the Mendelian reproduction, and individuals are competing against each other. First, in the absence of mutation, we focus on one gene and compute the fixation probability of an allele $a$ competing against a resident allele $A$ (Sections 3 and 4), as done in [2] for the simpler haploid case. We first consider the neutral case, where individuals all have the same birth, natural death, and competition death rates (i.e. alleles $A$ and $a$ are exchangeable). Here a martingale argument proves that the fixation probability of allele $a$ is simply equal to the initial proportion of this allele

Received 24 July 2012; revision received 8 April 2013.

* Current address: Université d'Orsay, Laboratoire de Mathématiques, 91405 Orsay Cedex, France.

Email address: camille.coron@math.u-psud.fr 
in the population. We then consider the case where allele $a$ is slightly nonneutral, i.e. natural death rates slightly deviate from the neutral case. Here we prove that the fixation probability of allele $a$ is differentiable in the parameters of deviation from the neutral case and that its partial derivatives are the unique subpolynomial solutions of Dirichlet problems. These equations consist of three-dimensional nonlinear double recurrence relationships which we manage to simplify to a one-dimensional double recurrence admitting a unique bounded solution. In Section 5 we add rare mutations and rescale time in order to observe mutation apparitions. At this time scale, mutations get fixed or disappear instantaneously, and the successive fixations of mutations are given by the jumps of a Markov process $S$ on the genotypes space, called the 'trait substitution sequence', introduced in [14] and notably studied in [1], and in [6] for the diploid case. Here the population size remains finite, and we do not use any deterministic approximation. Finally, we examine the successive jump rates of $S$ in the particular case of deleterious mutations (Section 5.3). We prove that, when every mutation is deleterious and if the birth rate is small enough, the Markov process $S$ jumps more and more rapidly. This means that the fixation rate of a deleterious mutation increases with the number of already fixed mutations, which creates a vicious circle called the extinction vortex (see [7] for biological interpretations and numerical results).

\section{Presentation of the model}

We consider a population of diploid hermaphroditic self-incompatible organisms, characterized by their genotypes. Building on the works of [1], [4], and [6], we consider a birthand-death process with mutation, selection, and competition under different time scales and we add diploidy. Each individual is characterized by its genotype $x \in \boldsymbol{G}:=\left\{\{\mathcal{A}, \mathcal{C}, \mathcal{G}, \mathcal{T}\}^{G}\right\}^{2}$, where $G$ is the genome size, and $\mathcal{A}, \mathcal{C}, \mathcal{G}$, and $\mathcal{T}$ are the four nucleotides that comprise DNA. Genotype $x=\left(x_{1}, x_{2}\right)$ is in fact composed of two DNA strands, $x_{1}$ and $x_{2}$, in $\{\mathcal{A}, \mathcal{C}, \mathcal{g}, \mathcal{T}\}^{G}$. In Sections 2 to 4 we consider the case without mutation and assume that the population is initially composed of individuals that only differ from each other on one gene. For this gene, there are two possible alleles, denoted by $A$ and $a$ in $\{\mathcal{A}, \mathcal{C}, \mathcal{g}, \mathcal{T}\}^{G^{\prime}}$, where $G^{\prime} \leq G$. The genotypes of individuals are thus denoted by $A A, A a$, and $a a$, and we represent the population dynamics by the Markov process

$$
Z: t \mapsto Z_{t}=\left(k_{t}, m_{t}, n_{t}\right)
$$

which gives the respective numbers of individuals with genotype $A A, A a$, and $a a$ at time $t$. For further simplicity, we will also refer to these genotypes as types 1,2 , and 3 . We assume that the process $Z$ is a birth-and-death process with competition on $\left(\mathbb{Z}_{+}\right)^{3}$, and we now detail the birth and death rates of the individuals of each genotype. The population has maximum fecundity rate $r$. More precisely, if the population contains $N$ individuals, $r N$ is the rate at which two distinct individuals of the population encounter one another, and the maximum total birth rate. These two individuals are chosen uniformly randomly in the population, and their encounter gives rise to a birth with a probability $p_{i j}\left(p_{i j}=p_{j i}\right)$ that depends on their two genotypes $i$ and $j$. The probability $p_{i j}$ can be defined biologically as the selective value associated with the genotypes $i$ and $j$, and represents both the degree of adaptation with respect to reproduction of types $i$ and $j$ and their compatibility. Finally, the new born individual results from a segregation (genetic melting between the genotypes of its parents), satisfying Mendel's laws of heredity. Then in the population $Z=(k, m, n)$ such that $k+m+n \geq 2$, if we define $b_{i j}:=r p_{i j}$, the 
rate $b_{i}(Z)$ at which an individual of type $i \in\{1,2,3\}$ arises is

$$
\begin{aligned}
& b_{1}(Z)=b_{11} \frac{k(k-1)}{N-1}+b_{12} \frac{k m}{N-1}+b_{22} \frac{m(m-1)}{4(N-1)}, \\
& b_{2}(Z)=b_{12} \frac{k m}{N-1}+b_{22} \frac{m(m-1)}{2(N-1)}+b_{23} \frac{m n}{N-1}+b_{13} \frac{2 k n}{N-1}, \\
& b_{3}(Z)=b_{33} \frac{n(n-1)}{N-1}+b_{23} \frac{m n}{N-1}+b_{22} \frac{m(m-1)}{4(N-1)} .
\end{aligned}
$$

Note that if the population $Z$ has size $N$,

$$
b_{1}(Z)+b_{2}(Z)+b_{3}(Z) \leq r N .
$$

We assume self-incompatibility, which implies that, when the population size reaches 1 , no further birth can occur and the population can be considered as extinct. Now individuals can die either naturally or due to competition with others. We denote by $d_{i}$ the natural death rate of individuals with type $i$ and by $c_{i j}$ the competition rate of $i$ against $j$, i.e. the rate at which a fixed individual of type $i$ makes a fixed individual of type $j$ die. We assume that

$$
c_{i j}>0 \text { for all } i, j \in\{1,2,3\}, \quad \text { i.e. } \quad \underline{c}=\inf _{i, j \in\{1,2,3\}} c_{i j}>0,
$$

and that, when the population size reaches 2 , no death can occur; hence, the population cannot become extinct. We then denote the state space of $Z$ by $\mathbb{N}_{* *}^{3}=\left(\mathbb{Z}_{+}\right)^{3} \backslash\{(0,0,0),(1,0,0),(0,1$, $0),(0,0,1)\}$. In the population $Z=(k, m, n)$ such that $k+m+n \geq 3$, the rate $d^{(i)}(Z)$ at which the population loses any individual of type $i$ is then

$$
\begin{aligned}
& d^{(1)}(Z)=\left(d_{1}+c_{11}(k-1)+c_{21} m+c_{31} n\right) k, \\
& d^{(2)}(Z)=\left(d_{2}+c_{12} k+c_{22}(m-1)+c_{32} n\right) m, \\
& d^{(3)}(Z)=\left(d_{3}+c_{13} k+c_{23} m+c_{33}(n-1)\right) n,
\end{aligned}
$$

and, if $k+m+n=2$,

$$
d^{(1)}(Z)=d^{(2)}(Z)=d^{(3)}(Z)=0 .
$$

From (2.2), (2.3), and Theorem 2.7.1 of [15], the process $Z$ does not explode. Then $Z_{t}$ is defined for all $t>0$, and we denote by $\mathbb{P}_{(k, m, n)}$ the law of $Z$ starting from state $(k, m, n)$, by $\mathbb{E}_{(k, m, n)}$ the associated expectation, by $\left(\mathcal{Z}_{l}\right)_{l \in \mathbb{Z}_{+}}$the embedded Markov chain, and by $\left(\mathcal{F}_{l}\right)_{l \in \mathbb{Z}_{+}}$ the filtration generated by $\mathcal{Z}$.

Notation. For every other process $X, \mathbb{P}_{X_{0}}^{X}$ is the law of $X$ starting from $X_{0}$, and $\mathbb{E}_{X_{0}}^{X}$ is the associated expectation. If $X$ is a continuous-time (respectively discrete-time) process, we denote by $T_{x}^{X}$ (respectively $\mathcal{T}_{x}^{X}$ ) the reaching time of $x$ by $X$.

In the following, the population size process will play a main role; we define $N: t \mapsto N_{t}=$ $\left(k_{t}+m_{t}+n_{t}\right)$, where $Z_{t}=\left(k_{t}, m_{t}, n_{t}\right)$ for every time $t>0$, and $\left(\mathcal{N}_{l}\right)_{l \in \mathbb{Z}_{+}}$to be the embedded Markov chain. $N$ is stochastically dominated by the logistic birth-and-death process $Y$ with transition rates

$$
a_{i j}= \begin{cases}r j & \text { if } j=i+1, \\ \underline{c} j(j-1) & \text { if } j=i-1 \text { and } i \neq 2, \\ 0 & \text { otherwise. }\end{cases}
$$

We define $y$ to be the embedded Markov chain. 
Proposition 2.1. For all $N \in \mathbb{N} \backslash\{1\}$, there exists $\rho>0$ such that $\mathbb{E}_{N}\left((1+\rho)^{\mathcal{T}_{2}^{y}}\right)<\infty$.

Proof. Let $N_{0}$ be such that $b<\left(d+c\left(N_{0}-1\right)\right)$. We assume that $N>N_{0}$, without loss of generality since $\mathbb{E}_{N^{\prime}}\left((1+\rho)^{\mathcal{T}_{2}^{y}}\right)<\mathbb{E}_{N}\left((1+\rho)^{\mathcal{T}_{2}^{y}}\right)$ if $N^{\prime}<N$. Note that it suffices to prove that, for every integer $n \in[3, N]$, there exists $\rho_{n}>0$ such that

$$
\mathbb{E}_{n}\left(\left(1+\rho_{n}\right)^{\left.T_{n-1}^{y}\right)<\infty} .\right.
$$

Indeed, by the strong Markov property, $\mathbb{E}_{N}\left((1+\rho)^{\mathcal{T}_{2}^{y}}\right)=\prod_{j=3}^{N} \mathbb{E}_{j}\left((1+\rho)^{T_{j-1}^{y}}<\infty\right.$ if $\rho \leq$ $\inf _{i \leq N} \rho_{i}$. Now, from [18, p. 428], (2.7) holds for $n=N$, since $N>N_{0}$. Now, following the proof of Lemma 5.11 of [5], let us prove by induction that if (2.7) holds for $n+1$ then it also holds for $n$. We assume that (2.7) holds for $n+1$ and that $Y_{0}=n$, and we define $M$ to be the random number of returns in $n$ before going to $n-1$. Here $M$ follows a geometrical law with parameter $p=b /(b+d+c(n-1))$. Then

$$
\mathcal{T}_{n-1}^{y}=M+1+\sum_{i=1}^{M} \mathcal{T}_{n, i}
$$

where the $\mathcal{T}_{n, i}$ are independent and distributed as $\mathcal{T}_{n}^{y}$ for all $i$. Then by the strong Markov property of the stopping times $\mathcal{T}_{n, i}$ we obtain

$$
\mathbb{E}_{n}\left((1+\rho)^{\mathcal{T}_{n-1}^{y}}\right) \leq \sum_{m=0}^{\infty}\left(\mathbb{E}_{n+1}\left((1+\rho)^{\mathcal{T}_{n}^{y}+2}\right)\right)^{m}(1-p) p^{m}
$$

Finally, since (2.7) holds for $n+1$, from the dominated convergence theorem, $\mathbb{E}_{n+1}((1+$ $\rho)^{\mathcal{T}_{n}^{y}+2}$ ) goes to 1 when $\rho$ goes to 0 ; hence, there exists $\rho_{n-1}$ such that $\mathbb{E}_{n+1}\left(\left(1+\rho_{n-1}\right)^{\mathcal{T}_{n}^{y}+2}\right)<$ $1 / p$, which gives the result.

Proposition 2.2. For all $p \geq 1$, if $\mathbb{E}\left(N_{0}^{p}\right)<\infty$ then $\sup _{t \geq 0} \mathbb{E}\left(N_{t}^{p}\right)<\infty$.

Proof. We set $Y_{0}=N_{0}$. It suffices to prove that $\sup _{t} \mathbb{E}\left(Y_{t}^{p}\right)<\infty$. The process $\left(Y_{t}\right)_{t>0}$ is a recurrent, irreducible, and ergodic Markov process on $\mathbb{N} \backslash\{1\}$, with stationary law $l$ (see (5.1) below for a more general case), and we can easily check that $E_{p}:=\sum_{j=2}^{\infty} l(j) j^{p}<\infty$ for all $p$. Now let us define the Markov process $\left(Y_{t}, Z_{t}\right)_{t \geq 0}$ such that $Y$ and $Z$ have the same transition rates, are independent, and $Z_{0}$ has law $l$. We define $\left(\mathcal{Y}_{n}, \mathcal{Z}_{n}\right)_{n \in \mathbb{Z}_{+}}$to be the associated Markov chain, and $\mathcal{T}=\inf \left\{n \mid \mathcal{Y}_{n}=\mathcal{Z}_{n}\right\}$. Following the proof of Theorem 6.6.4 of [10, p. 308], we have

$$
\begin{aligned}
\left|\mathbb{E}\left(\mathcal{y}_{n}^{p}\right)-E_{p}\right| & =\left|\mathbb{E}\left(y_{n}^{p}\right)-\mathbb{E}\left(\mathcal{Z}_{n}^{p}\right)\right| \\
& \leq \sum_{z \geq 2} z^{p}\left|\mathbb{P}\left(\mathcal{y}_{n}=z\right)-\mathbb{P}\left(\mathcal{Z}_{n}=z\right)\right| \\
& \leq \sum_{z \geq 2} z^{p}\left(\mathbb{P}\left(\mathcal{Y}_{n}=z, \mathcal{T}>n\right)+\mathbb{P}\left(\mathcal{Z}_{n}=z, \mathcal{T}>n\right)\right) \\
& =\mathbb{E}\left(\left(\mathcal{y}_{n}^{p}+\mathcal{Z}_{n}^{p}\right) \mathbf{1}_{\{\mathcal{T}>n\}}\right) \\
& \leq 2 \mathbb{E}\left(\mathcal{y}_{n}^{p} \mathbf{1}_{\{\mathcal{T}>n\}} \mathbf{1}_{\left\{Y_{0}>Z_{0}\right\}}\right)+2 \mathbb{E}\left(\mathcal{Z}_{n}^{p} \mathbf{1}_{\{\mathcal{T}>n\}} \mathbf{1}_{\left\{Z_{0}>Y_{0}\right\}}\right) \\
& \leq 2 \mathbb{E}\left(\mathcal{Y}_{n}^{p} \mathbf{1}_{\left\{\mathcal{T}_{2}{ }^{y}>n\right\}}\right)+2 \mathbb{E}\left(\mathcal{Z}_{n}^{p} \mathbf{1}_{\left\{\mathcal{T}_{2}^{\mathcal{Z}}>n\right\}}\right) .
\end{aligned}
$$


Now

$$
\begin{aligned}
\mathbb{E}\left(\mathcal{y}_{n}^{p} \mathbf{1}_{\left\{\mathcal{T}_{2}^{y}>n\right\}}\right) & \leq \sum_{z \geq 2}(z+n)^{p} \mathbb{P}\left(\mathcal{T}_{2}^{y}>n, Y_{0}=z\right) \\
& \leq 2^{p} \sum_{z \geq n} z^{p} \mathbb{P}\left(Y_{0}=z\right)+2^{p} \sum_{2 \leq z<n} n^{p} \mathbb{P}\left(\mathcal{T}_{2}^{y} \geq n, Y_{0}=z\right) \\
& \leq 2^{p} \sum_{z \geq n} z^{p} \mathbb{P}\left(Y_{0}=z\right)+2^{p} n^{p} \mathbb{P}\left(\mathcal{T}_{2}^{y} \geq n\right) .
\end{aligned}
$$

From Proposition 2.1, and since $\mathbb{E}\left(Y_{0}^{p}\right)<\infty, n^{p} \mathbb{P}\left(\mathcal{T}_{2}^{Y} \geq n\right)$ and $\sum_{z \geq n} z^{p} \mathbb{P}\left(Y_{0}=z\right)$ converge to 0 . Then $\mathbb{E}\left(Y_{n}^{p}\right)$ converges to $E_{p}$ when $n$ goes to $\infty$. Since $Y$ does not explode and $\mathbb{E}\left(Y_{0}^{p}\right)<$ $\infty$, we have $\sup _{t} \mathbb{E}\left(Y_{t}^{p}\right)<\infty$.

\section{Fixation probabilities}

\subsection{Absorbing states}

The birth-and-death process $Z$ admits the following absorbing state sets.

- $\Gamma_{a}=\{(0,0, n), n \geq 2\}$ is the set of states for which allele $a$ is fixed and allele $A$ has disappeared.

- $\Gamma_{A}=\{(k, 0,0), k \geq 2\}$ is the set of states for which allele $A$ is fixed and allele $a$ has disappeared.

- $\Gamma:=\Gamma_{a} \cup \Gamma_{A}$.

We are interested in computing the probability that allele $a$ goes to fixation (i.e. $Z$ reaches $\Gamma_{a}$ ), when $Z$ starts from any state $(k, m, n)$. We now define $\mathcal{T}_{\Omega}$ to be the (discrete) reaching time of set $\Omega$ by $\mathcal{Z}$ for all $\Omega \subset \mathbb{N}_{* *}^{3}$. The following result is an adaptation of Proposition 6.1 of [2] to the diploid case.

Proposition 3.1. There exists a constant $C$ such that, for any initial state $(k, m, n)$ in $\mathbb{N}_{* *}^{3}$, $\mathbb{E}_{(k, m, n)}\left(\mathcal{T}_{\Gamma}\right) \leq C(k+m+n)$ and $\mathbb{E}_{(k, m, n)}\left(\mathcal{T}_{\Gamma}^{2}\right) \leq C(k+m+n)^{2}$.

Proof. Let $\mathcal{T}_{\{2\}}$ be the first time the Markov chain $\mathcal{N}$ reaches 2 (or returns to 2 if $\mathcal{N}_{0}=2$ ), and define

$$
\overline{\mathcal{T}}_{\{2\} \rightarrow \Gamma}:=\sup _{(k, m, n) \mid k+m+n=2} \mathbb{E}_{(k, m, n)}\left(\mathcal{T}_{\Gamma}\right) .
$$

Then $\mathbb{E}_{(k, m, n)}\left(\mathcal{T}_{\Gamma}\right) \leq \mathbb{E}_{(k, m, n)}\left(\mathcal{T}_{\{2\}}\right)+\overline{\mathcal{T}}_{\{2\} \rightarrow \Gamma}$, and $\overline{\mathcal{T}}_{\{2\} \rightarrow \Gamma}$ is independent of $(k, m, n)$. We prove first that $\overline{\mathcal{T}}_{\{2\} \rightarrow \Gamma<\infty}<$ and then prove that there exists a constant $C_{1}$ such that $\mathbb{E}_{(k, m, n)}\left(\mathcal{T}_{\{2\}}\right)<C_{1}(k+m+n)$ for all $(k, m, n)$ in $\mathbb{N}_{* *}^{3}$. Now,

$$
\begin{aligned}
\overline{\mathcal{T}}_{\{2\} \rightarrow \Gamma} & =\sup _{(k, m, n) \mid k+m+n=2} \mathbb{E}_{(k, m, n)}\left(\mathcal{T}_{\Gamma} \mathbf{1}_{\left\{\mathcal{T}_{\{2\}} \geq \mathcal{T}_{\Gamma}\right\}}+\mathcal{T}_{\Gamma} \mathbf{1}_{\left\{\mathcal{T}_{\{2\}}<\mathcal{T}_{\Gamma}\right\}}\right) \\
& \leq \sup _{(k, m, n) \mid k+m+n=2} \mathbb{E}_{(k, m, n)}\left(\mathcal{T}_{\{2\}}\right)+\sup _{(k, m, n) \mid k+m+n=2} \mathbb{E}_{(k, m, n)}\left(\left(\mathcal{T}_{\Gamma}-\mathcal{T}_{\{2\}}\right) \mathbf{1}_{\left\{\mathcal{T}_{\{2\}}<\mathcal{T}_{\Gamma}\right\}}\right) \\
& \leq \sup _{(k, m, n) \mid k+m+n=2} \mathbb{E}_{(k, m, n)}\left(\mathcal{T}_{\{2\}}\right)
\end{aligned}
$$




$$
\begin{aligned}
& +\sup _{(k, m, n) \mid k+m+n=2} \sum_{\left(k^{\prime}, m^{\prime}, n^{\prime}\right) \mid k^{\prime}+m^{\prime}+n^{\prime}=2} \mathbb{E}_{(k, m, n)}\left(\left(\mathcal{T}_{\Gamma}-\mathcal{T}_{\{2\}}\right) \mathbf{1}_{\left\{\mathcal{T}_{\{2\}}<\mathcal{T}_{\Gamma}\right\}}\right. \\
& \times \sup _{(k, m, n) \mid k+m+n=2} \mathbb{E}_{(k, m, n)}\left(\mathcal{T}_{\{2\}}\right)+\overline{\mathcal{T}}_{\{2\} \rightarrow \Gamma} \sup _{(k, m, n) \mid k+m+n=2} \mathbb{P}_{(k, m, n)}\left(\left\{\mathcal{T}_{\{2\}}<\mathcal{T}_{\Gamma}\right\}\right),
\end{aligned}
$$

where the last inequality is obtained using the strong Markov property in $\mathcal{T}_{\{2\}}$. Defining

$$
\begin{gathered}
\bar{p}=\sup _{(k, m, n) \mid k+m+n=2} \mathbb{P}_{(k, m, n)}\left(\mathcal{T}_{\{2\}}<\mathcal{T}_{\Gamma}\right) \\
\text { and } \overline{\mathcal{T}}_{\{2\} \rightarrow\{2\}}=\sup _{(k, m, n) \mid k+m+n=2} \mathbb{E}_{(k, m, n)}\left(\mathcal{T}_{\{2\}}\right),
\end{gathered}
$$

we have $\bar{p}<1$, since, for every $(k, m, n)$ such that $k+m+n=2$, there exists a path for $Z$

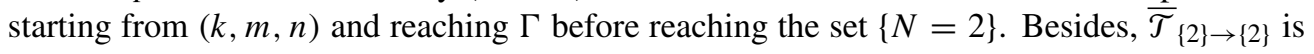
bounded by the expectation of the mean time for the embedded Markov chain $y$ defined by (2.6) to return to the set $\{N=2\}$. So $\overline{\mathcal{T}}_{\{2\} \rightarrow\{2\}}<\infty$, from Theorem 3.3.3 of [15]. Finally,

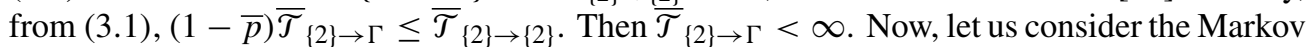
chain $\left(\mathcal{Y}_{n}\right)_{n \in \mathbb{Z}_{+}}$on $\mathbb{N} \backslash\{1\}$, associated with $Y$. Since $\mathcal{N}$ is stochastically dominated by $\mathcal{Y}$, if $N=k+m+n, \mathbb{E}_{(k, m, n)}\left(\mathcal{T}_{\{2\}}\right) \leq \mathbb{E}_{N}^{y}\left(\inf \left\{n \mid \mathcal{y}_{n}=2\right\}\right)$. Define $S_{N, i}=\mathbb{E}_{N}^{y}\left(\inf \left\{n \mid \mathcal{y}_{n}=i\right\}\right)$, and let $N_{0} \geq 2$ be a natural integer such that $\bar{b} /\left(\bar{b}+\underline{c} N_{0}\right) \leq \frac{1}{3}$. If $N \geq N_{0}$ then $S_{N, 2}=$ $S_{N, N_{0}}+S_{N_{0}, 2}$. Moreover, since $\bar{b} /(\bar{b}+\underline{c} N) \leq \frac{1}{3}$ for all $N \geq N_{0}, S_{N, N_{0}} \leq \mathbb{E}\left(U_{N, N_{0}}\right)$, where $U_{N, i}$ is the first reaching time of $i$ for the discrete-time random walk on $\mathbb{Z}$ that starts from $N$ and has probability $\frac{1}{3}$ of jumping one step to the right and probability $\frac{2}{3}$ of jumping one step to the left for every state. We know that $\mathbb{E}\left(U_{N, N_{0}}\right)=3\left(N-N_{0}\right)$ [15, pp. 21-22]. So, if $N \geq N_{0}, S_{N, 2} \leq S_{N_{0}, 2}+3\left(N-N_{0}\right)$ and $S_{N_{0}, 2}<\infty$ from Proposition 2.1. Then there exists a constant $C_{1}>0$ such that $S_{N, 2}<C_{1} N$ for all $N \geq 2$. Similarly, as $\mathbb{E}\left(U_{N, N_{0}}^{2}\right)=$ $3\left(N-N_{0}\right)\left(3\left(N-N_{0}\right)+8\right)\left[15\right.$, pp. 21-22], we prove that $\mathbb{E}_{(k, m, n)}\left(\mathcal{T}_{\Gamma}\right) \leq C(k+m+n)^{2}$.

We now consider the fixation probabilities of allele $a$ as a function of the initial state of the population. Let $F_{a}=\left\{\left(Z_{t}\right)_{t>0}\right.$ reaches $\left.\Gamma_{a}\right\}$, and define $u(Z)=\mathbb{E}_{Z}\left(\mathbf{1}_{F_{a}}\right)$ to be the fixation probability of allele $a$, knowing that the population starts from state $Z ; u$ also depends on the demographic parameters of the population, and this dependence will be explicitly written down when necessary. Note that $\left(u\left(Z_{t}\right)\right)_{t>0}$ is a martingale since

$$
u\left(Z_{t}\right)=u\left(k_{t}, m_{t}, n_{t}\right)=\mathbb{E}_{Z_{t}}\left(\mathbf{1}_{F_{a}}\right)=\mathbb{E}\left(\mathbf{1}_{F_{a}} \mid \mathcal{F}_{t}\right) .
$$

In the neutral case (Section 3.2), a martingale argument gives the value of $u$; in the nonneutral case with small mutation assumption (Section 3.3), we prove that $u$ admits a Taylor expansion in the parameters of deviation from the neutral case.

\subsection{Neutral case}

We now consider the neutral case when ecological parameters do not depend on genotypes, i.e. when $b_{i j}=b, c_{i j}=c$, and $d_{i}=d$ for all $i$ and $j$ in $\{1,2,3\}$. We first prove the following result.

Proposition 3.2. In the neutral case, for all $(k, m, n)$ in $\mathbb{N}_{* *}^{3}$ and all ecological parameters $b$, $d$, and $c$,

$$
u(k, m, n)=\frac{m+2 n}{2(k+m+n)} .
$$


Proof. Let us define the function $p:(k, m, n) \mapsto(m+2 n) / 2(k+m+n)$ and denote by $T_{l}$ the $l$ th jump time of the population (i.e. the time at which the $l$ th event, a birth or death, occurs). The Markov chain $\left(p\left(\mathcal{Z}_{l}\right)\right)_{l \in \mathbb{Z}_{+}}$gives the successive proportions of allele $a$ in the population. We now prove that $\left(p\left(\mathcal{Z}_{l}\right)\right)_{l \in \mathbb{Z}_{+}}$is an $\mathscr{F}_{l}$-bounded martingale. To this end, we distinguish two types of state: one in which the population size is greater than or equal to three and another in which it is equal to two. For $\mathcal{Z}_{l}=\left(k_{l}, m_{l}, n_{l}\right)$ such that $\mathcal{N}_{l} \geq 3$, we can compute $\mathbb{E}\left(p\left(\mathcal{Z}_{l+1}\right) \mid \mathcal{Z}_{l}\right)$ by decomposing it according to the nature of the $(l+1)$ th event:

$$
\begin{aligned}
& \mathbb{E}\left(p\left(\mathbb{Z}_{l+1}\right) \mid \mathcal{Z}_{l}\right)=\frac{2 \mathcal{N}_{l} p\left(\mathcal{Z}_{l}\right)-2}{2 \mathcal{N}_{l}-2} \mathbb{P}(\text { death of } a a)+\frac{2 \mathcal{N}_{l} p\left(Z_{l}\right)-1}{2 \mathcal{N}_{l}-2} \mathbb{P}(\text { death of } A a) \\
& +\frac{2 \mathcal{N}_{l} p\left(\mathcal{Z}_{l}\right)}{2 \mathcal{N}_{l}-2} \mathbb{P}(\text { death of } A A)+\frac{2 \mathcal{N}_{l} p\left(\mathcal{Z}_{l}\right)+2}{2 \mathcal{N}_{l}+2} \mathbb{P}(\text { birth of } a a) \\
& +\frac{2 \mathcal{N}_{l} p\left(\mathcal{Z}_{l}\right)+1}{2 \mathcal{N}_{l}+2} \mathbb{P}(\text { birth of } A a)+\frac{2 \mathcal{N}_{l} p\left(\mathcal{Z}_{l}\right)}{2 \mathcal{N}_{l}+2} \mathbb{P}(\text { birth of } A A) \\
& =p\left(\mathbb{Z}_{l}\right) \text {. }
\end{aligned}
$$

The same result can be easily proved for $N_{l}=2$. From Doob's stopping time theorem applied to the bounded martingale $\left(p\left(Z_{l}\right)\right)_{l}$ and to the stopping time $\mathcal{T}_{\Gamma}$ (almost surely finite, from Proposition 3.1), we obtain

$$
\mathbb{E}_{k, m, n}\left(p\left(\mathcal{Z}_{\mathcal{T}_{\Gamma}}\right)\right)=\frac{2 n+m}{2(k+m+n)} .
$$

Now

$$
\begin{aligned}
\mathbb{E}_{k, m, n}\left(p\left(\mathcal{Z}_{\mathcal{T}_{\Gamma}}\right)\right) & =\mathbb{E}_{k, m, n}\left(p\left(\mathcal{Z}_{\mathcal{T}_{\Gamma}}\right) \mathbf{1}_{\left\{T_{\Gamma_{a}}<T_{\Gamma_{A}}\right\}}\right)+\mathbb{E}_{k, m, n}\left(p\left(\mathcal{Z}_{\mathcal{T}_{\Gamma}}\right) \mathbf{1}_{\left\{T_{\Gamma_{a}}>T_{\Gamma_{A}}\right\}}\right) \\
& =\mathbb{P}_{k, m, n}\left(T_{\Gamma_{a}}<T_{\Gamma_{A}}\right) \\
& =u(k, m, n)
\end{aligned}
$$

since $\mathbb{E}_{k, m, n}\left(p\left(\mathcal{Z}_{{\widetilde{\tau_{\Gamma}}}}\right) \mid T_{\Gamma_{a}}<T_{\Gamma_{A}}\right)=1$ and $\mathbb{E}_{k, m, n}\left(p\left(\mathcal{Z}_{\mathcal{T}_{\Gamma}}\right) \mid T_{\Gamma_{a}}>T_{\Gamma_{A}}\right)=0$.

When the mutation is not neutral, we do not obtain any closed formula for $p(Z)$ as previously. We instead consider the Dirichlet problem satisfied by $u$.

\subsection{Deviation from the neutral case}

3.3.1. A Dirichlet problem. We now arbitrarily assume that allele $a$ is slightly deleterious, i.e. the demographic parameters $\left(b_{i j}\right)_{i, j},\left(c_{i j}\right)_{i, j}$, and $\left(d_{i}\right)_{i}$ are less advantageous for genotypes $A a$ and $a a$ than for genotype $A A$, and slightly deviate from the neutral case. This latter assumption (small mutation sizes) is justified in biology papers, such as [16] and [17], by showing that species evolution is partly due to the fixation of a large number of small mutations. Besides, we assume that carrying allele $a$ only influences the natural death rate of individuals. More precisely, we set

$$
\begin{aligned}
& \quad b_{i j}=b \text { for all } i, j, \quad c_{i j}=c \text { for all } i, j, \\
& \text { whereas } d_{1}=d, \quad d_{2}=d+\delta, \quad \text { and } d_{3}=d+\delta^{\prime},
\end{aligned}
$$

where $\delta$ and $\delta^{\prime}$ are close to 0 . Note that if $\delta^{\prime}$ is positive and $\delta$ is equal to 0 , then allele $a$ is deleterious. The effect of $\delta$ is more intricate because it affects heterozygous individuals, with the same apparent effect on both alleles. It simply represents a more or less important adaptation of heterozygotes compared to $A A$ homozygotes and, as we will see later (Subsection 3.3.2), its 
role in the deleterious or positive effect of allele $a$ depends on the initial genetic repartition of the population. We denote by $L^{\delta, \delta^{\prime}}$ the infinitesimal generator of $Z$ that satisfies the conditions in (3.3), and by $u\left((k, m, n), \delta, \delta^{\prime}\right)$ the fixation probability of allele $a$, knowing that $Z$ starts from $(k, m, n)$ for all $(k, m, n)$ in $\mathbb{N}_{* *}^{3}$. We then have, for all real bounded functions $f$ on $\mathbb{N}_{* *}^{3}$,

$$
\begin{aligned}
\left(L^{\delta, \delta^{\prime}} f\right)(k, m, n)= & b_{1}(Z) f(k+1, m, n)+b_{2}(Z) f(k, m+1, n)+b_{3}(Z) f(k, m, n+1) \\
& +d^{(1)}(Z) f(k-1, m, n)+d^{(2)}(Z) f(k, m-1, n) \\
& +d^{(3)}(Z) f(k, m, n-1) \\
& -\left(b N+(d+c(N-1)) N+\delta m+\delta^{\prime} n\right) f(k, m, n) .
\end{aligned}
$$

From (2.1), (2.4), and (2.5), we define the infinitesimal generator

$$
\begin{aligned}
& (L v)(k, m, n)=\left(L^{0,0} v\right)(k, m, n) \\
& =\frac{b}{N-1}\left[\left(k(k-1)+k m+\frac{m(m-1)}{4}\right) v(k+1, m, n)\right. \\
& +\left(k m+\frac{m(m-1)}{2}+m n+2 k n\right) v(k, m+1, n) \\
& \left.+\left(n(n-1)+m n+\frac{m(m-1)}{4}\right) v(k, m, n+1)\right] \\
& +(d+c(N-1))[k v(k-1, m, n)+m v(k, m-1, n)+n v(k, m, n-1)] \\
& -(b N+d N+c N(N-1)) v(k, m, n) \text { if } k+m+n \geq 3 \text {, } \\
& (L v)(k, m, n)=\frac{b}{N-1}\left[\left(k(k-1)+k m+\frac{m(m-1)}{4}\right) v(k+1, m, n)\right. \\
& +\left(k m+\frac{m(m-1)}{2}+m n+2 k n\right) v(k, m+1, n) \\
& \left.+\left(n(n-1)+m n+\frac{m(m-1)}{4}\right) v(k, m, n+1)\right] \\
& -b N v(k, m, n) \quad \text { if } k+m+n=2 \text {. }
\end{aligned}
$$

Using the fact that $\left(u\left(Z_{t}, \delta, \delta^{\prime}\right)\right)_{t \leq 0}$ is a bounded martingale if $Z$ has infinitesimal generator $L^{\delta, \delta^{\prime}}$ (3.2), we obtain the following result.

Proposition 3.3. $u\left(\cdot, \delta, \delta^{\prime}\right)$ satisfies

$$
\begin{gathered}
\left(L^{\delta, \delta^{\prime}} u\left(\cdot, \delta, \delta^{\prime}\right)\right)(k, m, n)=0 \quad \text { for all }(k, m, n) \mid N=k+m+n \geq 2, \\
u\left((0,0, n), \delta, \delta^{\prime}\right)=1 \quad \text { for all } n \geq 2, \\
u\left((k, 0,0), \delta, \delta^{\prime}\right)=0 \text { for all } k \geq 2
\end{gathered}
$$

Our main result in this section is the following theorem, which details the deviation of $u$ from the neutral case.

Theorem 3.1. For all $(k, m, n)$ in $\mathbb{N}_{* *}^{3}$, the function $\left(\delta, \delta^{\prime}\right) \mapsto u\left((k, m, n), \delta, \delta^{\prime}\right)$ is an analytic function of $\left(\delta, \delta^{\prime}\right)$ in the neighborhood of $(0,0)$. Moreover, if we set $Y=2 k+m$,

$$
u\left((k, m, n), \delta, \delta^{\prime}\right)=p(k, m, n)-\delta v(k, m, n)-\delta^{\prime} v^{\prime}(k, m, n)+o\left(|\delta|+\left|\delta^{\prime}\right|\right),
$$


where

$$
\begin{aligned}
v(k, m, n) & =(k-n)\left[\frac{m}{N} x_{N}+\frac{N^{2}-(k-n)^{2}}{N^{2}} y_{N}\right] \\
v^{\prime}(k, m, n) & =\frac{n Y}{N} x_{N}+m x_{N}^{\prime}+Y(2 N-Y)\left(\frac{y_{N}^{\prime}}{N}-\frac{Y}{2 N^{2}} y_{N}\right) .
\end{aligned}
$$

The sequences $x_{N}, y_{N}, x_{N}^{\prime}$, and $y_{N}^{\prime}$ are defined as the unique bounded solutions of second-order recurrence equations (Propositions 3.5 and 3.6).

The proof of this theorem is decomposed into several parts: the existence and formula of the two partial derivatives are obtained in Sections 3.3.2 and 3.3.3 and the analyticity of $u$ is given in Section 4.5. In the following two subsections, we separately consider the cases in which $\delta=0$ and $\delta^{\prime}=0$.

3.3.2. The dependence of $u$ in $\delta$. To simplify the notation, we define $u((k, m, n), \delta)=u((k, m$, $n), \delta, 0)$. We will show that the derivative of $u$ at $\delta=0$ is the unique subpolynomial (i.e. lower than a polynomial function in $N=k+m+n$ ) solution of a nonlinear recurrence equation in $(k, m, n)$. Such a result has been obtained in [2] for the haploid case. Here the nonlinearity due to both the competition and diploid segregation terms generates new mathematical difficulties. We will use some arguments developed in [2], focusing on the difficulties caused by diploidy. We say that a function $f$ on $\left(\mathbb{Z}_{+}\right)^{3}$ is sublinear if there exists a constant $C$ such that $|f(k, m, n)| \leq$ $C(k+m+n)$ for every $(k, m, n)$.

Proposition 3.4. For all $(k, m, n)$ in $\mathbb{N}_{* *}^{3}, u((k, m, n), \cdot)$ is differentiable at 0 . Its derivative $v(k, m, n)$ is the unique sublinear solution of the system of equations

$$
\begin{gathered}
(L v)(k, m, n)=\frac{m(n-k)}{2 N(N-1)} \text { for all }(k, m, n) \mid k+m+n \geq 3, \\
(L v)(k, m, n)=0 \text { for all }(k, m, n) \mid k+m+n=2, \\
v(2,0,0)=v(0,0,2)=0 .
\end{gathered}
$$

Proof. The differentiability is implied by the analyticity of $u$, claimed in Theorem 3.1 and proved in Subsection 4.5. For the sublinearity of the derivative, as in the simplest case of haploid populations, we introduce paths of $Z$, i.e. the sequence of states visited by this process. Indeed the fixation probability of the mutant allele $a$ if the population $Z$ starts from state $(k, m, n)$ can be written as the sum of the probabilities of every path starting from $(k, m, n)$ and reaching a state $\left(0,0, n^{\prime}\right)$ with $n^{\prime} \geq 2$. We then denote by $S_{(k, m, n) \rightarrow \Omega}$ the set of paths linking $(k, m, n) \notin \Gamma$ to $\Omega$ without reaching $\Gamma$ before $\Omega$, and by $\left(i_{1}, i_{2}, \ldots, i_{l}\right)$ a path, where $i_{j}$ is the $j$ th state of the path. We finally denote by $\pi_{i_{j} i_{j+1}}^{\delta}$ the transition probability from state $i_{j}$ to state $i_{j+1}$ for $Z$. Then

$$
u((k, m, n), \delta)=\sum_{\left(i_{1}, \ldots, i_{l}\right) \in S_{(k, m, n) \rightarrow \Gamma_{a}}} \pi_{i_{1} i_{2}}^{\delta} \cdots \pi_{i_{l-1} i_{l}}^{\delta} .
$$

Now $\pi_{i_{j} i_{j+1}}^{\delta}$ is a differentiable function of $\delta$ and the absolute value of its derivative at $\delta=0$ is bounded independently of $(k, m, n)$ by a constant denoted by $C_{1}$. To prove this latter assertion, we separately consider the different possible transitions for the population in state $(k, m, n)$. For instance, the transition probability from state $(k, m, n)$ to state $(k+1, m, n)$ is

$$
\pi_{(k, m, n)(k+1, m, n)}^{\delta}=\frac{b(k(k-1)+k m+m(m-1) / 4)}{(N-1)(b N+d N+\delta m+c N(N-1))} .
$$


Then $\pi_{(k, m, n)(k+1, m, n)}^{\delta}$ is differentiable with respect to $\delta$ at 0 , and

$$
\begin{aligned}
\left|\frac{\partial \pi_{(k, m, n)(k+1, m, n)}^{\delta}}{\partial \delta}\right|_{\delta=0} \mid & =\frac{m b(k(k-1)+k m+m(m-1) / 4)}{(N-1)(b N+d N+c N(N-1))^{2}} \\
& \leq \frac{m}{b N+d N+c N(N-1)} \\
& \leq \frac{2}{b+d+2 c} .
\end{aligned}
$$

Similar computations are made for other possible transitions. Then $u_{(k, m, n)}^{\delta}$ is differentiable with respect to $\delta$ at $\delta=0$ (Theorem 3.1) and

$$
\begin{aligned}
& \left|\frac{\partial u((k, m, n), \delta)}{\partial \delta}\right|_{\delta=0} \mid \\
& =\left.\sum_{\left(i_{1}, \ldots, i_{l}\right) \in S_{(k, m, n) \rightarrow \Gamma a}} \sum_{l^{\prime}=1}^{l-1} \pi_{i_{1} i_{2}}^{0} \cdots \pi_{i_{l^{\prime}-1} i_{l}^{\prime}}^{0} \frac{\partial \pi_{i_{l^{\prime}} i_{l^{\prime}+1}}^{\delta}}{\partial \delta}\right|_{\delta=0} \pi_{i_{1^{\prime}+1} i_{l^{\prime}+2}}^{0} \cdots \pi_{i_{l-1} i_{l}}^{0} \\
& \leq C_{1} \sum_{l^{\prime} \geq 1} \sum_{\left(k^{\prime}, m^{\prime}, n^{\prime}\right) \in \mathbb{N}_{* *}^{3} \backslash \Gamma} \sum_{\left(i_{1}, \ldots, i_{l^{\prime}}\right) \in S_{(k, m, n) \rightarrow\left(k^{\prime}, m^{\prime}, n^{\prime}\right)}} \pi_{i_{1} i_{2}}^{0} \cdots \pi_{i_{l^{\prime}-1} i_{l}^{\prime}}^{0} \\
& \times \sum_{\varepsilon \in \mathbb{Z}^{3},\|\varepsilon\|=1} \sum_{l^{\prime \prime} \geq 0,\left(j_{1}, \ldots, j_{l^{\prime \prime}}\right) \in S_{\left(k^{\prime}, m^{\prime}, n^{\prime}\right)+\varepsilon \rightarrow \Gamma_{a}}} \pi_{j_{1} j_{2}}^{0} \cdots \pi_{j_{l^{\prime \prime}-1} j_{l^{\prime \prime}}}^{0} .
\end{aligned}
$$

Then

$$
\begin{aligned}
|v(k, m, n)| \leq & C_{1} \sum_{l^{\prime} \geq 1} \sum_{\left(k^{\prime}, m^{\prime}, n^{\prime}\right) \in \mathbb{N}_{* *}^{3} \backslash \Gamma} \sum_{\left(i_{1}, \ldots, i_{l^{\prime}}\right) \in S_{(k, m, n) \rightarrow\left(k^{\prime}, m^{\prime}, n^{\prime}\right)}} \pi_{i_{1} i_{2}}^{0} \cdots \pi_{i_{l^{\prime}-1} i_{l}^{\prime}}^{0} \\
& \times \sum_{\varepsilon \in \mathbb{Z}^{3},\|\varepsilon\|=1} \mathbb{P}_{\left(k^{\prime}, m^{\prime}, n^{\prime}\right)+\varepsilon}\left(T_{\Gamma_{a}}<T_{\Gamma_{A}}\right) \\
\leq & 6 C_{1} \sum_{l^{\prime} \geq 1} \mathbb{P}_{(k, m, n)}\left(\mathcal{T}_{\Gamma}>l^{\prime}\right) \quad \text { (the latter sum being lower than 6) } \\
= & 6 C_{1} \mathbb{E}_{(k, m, n)}\left(\mathcal{T}_{\Gamma}-1\right) .
\end{aligned}
$$

From Proposition 3.1, $\mathbb{E}_{(k, m, n)}\left(\mathcal{T}_{\Gamma}\right)<C_{2}(k+m+n)$ for a constant $C_{2}$, from which it follows that $u((k, m, n), \cdot)$ is differentiable with respect to $\delta$ and that its derivative at $0, v(k, m, n)$, is sublinear. Now, identifying the first-order terms in $\delta$ in (3.5), we see that $v$ satisfies, for all $(k, m, n) \in \mathbb{N}_{* *}^{3}$,

$$
\begin{gathered}
(L v)(k, m, n)=\frac{m(n-k)}{2 N(N-1)} \text { for all }(k, m, n) \mid k+m+n \geq 3, \\
(L v)(k, m, n)=0 \quad \text { for all }(k, m, n) \mid k+m+n=2, \\
v(2,0,0)=v(0,0,2)=0 .
\end{gathered}
$$

It remains to prove that the system of equations (3.8) admits a unique subpolynomial solution. Let $h$ be a subpolynomial solution of the equation $L h=0$ such that $h(2,0,0)=h(0,0,2)=0$. Then $\left(h\left(\mathcal{Z}_{l}\right)\right)_{l \in \mathbb{Z}_{+}}$is an $\mathcal{F}_{l}$-martingale if $\mathcal{Z}$ is the neutral Markov chain associated to the birthand-death process with generator $L$. On $\Gamma_{A}$, the equation $L h(k, m, n)=0$ gives, for all 
$k \geq 3$,

$$
\begin{gathered}
b k(h(k+1,0,0)-h(k, 0,0))=(d k+c k(k-1))(h(k, 0,0)-h(k-1,0,0)), \\
\text { i.e. } \alpha_{k+1}=\frac{d k+c k(k-1)}{b k} \alpha_{k}
\end{gathered}
$$

if $\alpha_{k}=h(k, 0,0)-h(k-1,0,0)$ for all $k \geq 3$. Since $h$ is subpolynomial, necessarily $\alpha_{3}=0$, and, therefore, since $h(2,0,0)=0, h \equiv 0$ on $\Gamma_{A}$. Similarly, $h \equiv 0$ on $\Gamma_{a}$. Besides, there exists a positive integer $q$ such that

$$
\sup _{t} \mathbb{E}_{k, m, n}\left(\left|h\left(Z_{t}\right)\right|^{2}\right) \leq \sup _{t} \mathbb{E}_{k, m, n}\left(C\left|k_{t}+m_{t}+n_{t}\right|^{2 q}\right) .
$$

Moreover, from Proposition 2.2, $\sup _{t} \mathbb{E}_{k, m, n}\left(\left|k_{t}+m_{t}+n_{t}\right|^{2 q}\right)<+\infty$ for all $(k, m, n)$ in $\mathbb{N}_{* *}^{3}$. Then the martingale $\left(h\left(\mathbb{Z}_{l}\right)\right)_{l \in \mathbb{Z}_{+}}$is uniformly integrable. From Doob's stopping time theorem applied to the stopping time $\mathcal{T}_{\Gamma}$, we then have $0=\mathbb{E}_{k, m, n}\left(h\left(\mathcal{Z}_{\mathcal{T}_{\Gamma}}\right)\right)=h(k, m, n)$.

Let us now state the following proposition whose proof is given in Section 4.

Proposition 3.5. For all $(k, m, n)$ such that $k+m+n \geq 2$,

$$
v(k, m, n)=(k-n)\left[\frac{m}{N} x_{N}+\frac{N^{2}-(k-n)^{2}}{N^{2}} y_{N}\right],
$$

where the sequence of vectors

$$
\left(z_{N}\right)_{N \geq 3}=\left(\begin{array}{l}
x_{N} \\
y_{N}
\end{array}\right)_{N \geq 3}
$$

is the unique subpolynomial solution of the system of equations

$$
\begin{gathered}
B_{N} z_{N+1}=C_{N} z_{N}+D_{N} z_{N-1}+f_{N} \quad \text { for all } N \geq 4, \\
B_{3} z_{4}=\tilde{C}_{3} z_{3}+f_{3},
\end{gathered}
$$

with

$$
\begin{aligned}
& B_{N}:=\frac{b}{2(N-1)(N+1)}\left(\begin{array}{cc}
1 & \frac{2 N^{2}+4 N-3}{N+1} \\
2 N^{2}-3 & \frac{-3}{N+1}
\end{array}\right), \\
& C_{N}:=(b+d+c(N-1))\left(\begin{array}{cc}
0 & \frac{1}{N} \\
1 & 0
\end{array}\right) \text {, } \\
& \tilde{C}_{3}:=\left(\begin{array}{cc}
0 & \frac{b+d+2 c}{3} \\
b+\frac{d+2 c}{3} & -(d+2 c)
\end{array}\right)=C_{3}-\left(\begin{array}{cc}
0 & 0 \\
\frac{2}{3}(d+2 c) & (d+2 c)
\end{array}\right) \text {, } \\
& D_{N}:=-\frac{d+c(N-1)}{N-1}\left(\begin{array}{cc}
0 & \frac{N-3}{N-1} \\
N-2 & \frac{3}{N-1}
\end{array}\right) \text {, } \\
& f_{N}:=\left(\begin{array}{c}
0 \\
\frac{-1}{2 N(N-1)}
\end{array}\right) \text {. }
\end{aligned}
$$


Note that here $v(k, m, n)=-v(n, m, k)$ and that the comparison between the proportions of genotypes $A A$ and $a a$ play a particular role in the value and sign of $v$.

3.3.3. The dependence of $u$ in $\delta^{\prime}$. In this section we set $\delta=0$, i.e. $a$ is a recessive allele, and deleterious when $\delta^{\prime}>0$. As in the previous section (Proposition 3.4), $u_{k, m, n}^{0, \cdot}: \delta^{\prime} \mapsto u^{0, \delta^{\prime}}$ is differentiable and $v^{\prime}$ is the unique sublinear solution of the system

$$
\begin{gathered}
L v^{\prime}(k, m, n)=\frac{n Y}{2 N(N-1)} \text { for all }(k, m, n) \mid k+m+n \geq 3, \\
L v^{\prime}(k, m, n)=0 \quad \text { for all }(k, m, n) \mid k+m+n=2, \\
v^{\prime}(2,0,0)=v^{\prime}(0,0,2)=0
\end{gathered}
$$

where $Y=2 k+m$ is the number of $A$ alleles in the population $(k, m, n)$.

The following proposition (proved in Subsection 4.4) gives a formula for $v^{\prime}(k, m, n)$.

Proposition 3.6. It holds that

$$
v^{\prime}(k, m, n):=\frac{n Y}{N} x_{N}+m x_{N}^{\prime}+Y(2 N-Y)\left(\frac{y_{N}^{\prime}}{N}-\frac{Y}{2 N^{2}} y_{N}\right),
$$

where $x_{N}$ and $y_{N}$ are defined in Proposition 3.5, and the sequence of vectors

$$
z_{N}^{\prime}=\left(\begin{array}{c}
x_{N}^{\prime} \\
y_{N}^{\prime}
\end{array}\right)
$$

is the unique subpolynomial solution of the system of equations

$$
\begin{gathered}
B_{N}^{\prime} z_{N+1}^{\prime}=C_{N}^{\prime} z_{N}^{\prime}+D_{N}^{\prime} z_{N-1}^{\prime}+f_{N}^{\prime} \quad \text { for all } N \geq 3, \\
\tilde{B}_{2}^{\prime} z_{3}^{\prime}=\tilde{C}_{2}^{\prime} z_{2}^{\prime}+\tilde{f}_{2}^{\prime},
\end{gathered}
$$

with

$$
\begin{aligned}
B_{N}^{\prime} & :=\frac{b}{N-1}\left(\begin{array}{cc}
2 N^{2}-2 N-1 & \frac{-1}{N+1} \\
\frac{1}{2} & \frac{N^{2}+N-3 / 2}{N+1}
\end{array}\right) \\
\tilde{B}_{2}^{\prime} & :=\left(\begin{array}{cc}
1 & 3 \\
3 & \frac{13}{3}
\end{array}\right) \\
C_{N}^{\prime} & :=(b N+d N+c N(N-1))\left(\begin{array}{cc}
2 & 0 \\
0 & \frac{1}{N}
\end{array}\right) \\
\tilde{C}_{2}^{\prime} & :=\left(\begin{array}{ll}
0 & 2 \\
2 & 3
\end{array}\right), \\
D_{N}^{\prime} & :=-(d+c(N-1))\left(\begin{array}{cc}
2 N-2 & \frac{2}{N-1} \\
0 & \frac{N-2}{N-1}
\end{array}\right)
\end{aligned}
$$




$$
\begin{aligned}
& f_{N}^{\prime}:=\left(\begin{array}{c}
\frac{b}{N-1}(2 N-1) \frac{y_{N+1}}{2(N+1)^{2}}-(d+c(N-1))(4 N+2) \frac{y_{N-1}}{2(N-1)^{2}} \\
\frac{b}{N-1}\left(2 N^{3}+3 N^{2}-4 N-\frac{3}{2}\right) \frac{y_{N+1}}{2(N+1)^{2}} \\
-(b N+d N+c N(N-1))(2 N-1) \frac{y_{N}}{2 N^{2}} \\
+(d+c(N-1))\left(2 N^{2}-7 N+8\right) \frac{y_{N-1}}{2(N-1)^{2}}
\end{array}\right), \\
& \tilde{f}_{2}^{\prime}:=\left(\begin{array}{c}
x_{2}-y_{2}-x_{3}+\frac{3}{2} y_{3} \\
\frac{19}{6} y_{3}-\frac{9}{4} y_{2}
\end{array}\right) .
\end{aligned}
$$

We now prove Propositions 3.5 and 3.6. In both cases, the proof is divided into two parts: we first prove the result when the fecundity $b$ is small enough compared to the competition parameter $c$, and then we generalize the result to all possible demographic parameters $b, d$, and $c$.

\section{Proofs of Propositions 3.5 and 3.6}

\subsection{Proof of Proposition 3.5 for small $b$}

To begin with, calculations give the following lemma.

Lemma 4.1. (i) If(3.6) holds then $v$ satisfies (3.8) if and only if $\left(z_{N}\right)_{N \geq 3}$ satisfies (3.10), (3.11), and $x_{2}+\frac{3}{2} y_{2}=\frac{4}{3} x_{3}+2 y_{3}$.

(ii) $(v(k, m, n))_{(k, m, n) \in \mathbb{N}_{* *}^{3}}$ is sublinear if and only if $\left(z_{N}\right)_{N \geq 3}$ is bounded.

Proof. Part (ii) is easy to see from (3.6). For part (i), if $v$ satisfies (3.6) then from the second equation of (3.8) we easily obtain $x_{2}+\frac{3}{2} y_{2}=\frac{4}{3} x_{3}+2 y_{3}$. Now to derive (3.10) from the first equation of (3.8), we have to write the birth and death rates given in (2.1) and (2.4) as functions of the variables $m, k-n$, and $N$. Then we separate terms with $x_{N}, y_{N}, x_{N+1}, y_{N+1}, x_{N-1}$, and $y_{N-1}$. Each of these terms is multiplied by the sum of two polynomial functions: one of the form $m(k-n) P(N)$ and the other of the form $(k-n)\left(N^{2}-(k-n)^{2}\right) Q(N)$, where $P$ and $Q$ are polynomial functions. The total term that multiplies the polynomial function $(k-n)\left(N^{2}-(k-n)^{2}\right)$ must be equal to 0 , while the term that multiplies the polynomial function $m(k-n)$ must be equal to $-1 / 2 N(N-1)$ from (3.8). We deduce from this that (3.10) is in fact true for all $N \geq 3$. Finally, the initial condition (3.11) is obtained by combining (3.10) for all $N=3$ and the relation $x_{2}+\frac{3}{2} y_{2}=\frac{4}{3} x_{3}+2 y_{3}$. Conversely, if $\left(z_{N}\right)_{N \geq 3}$ satisfies (3.10), (3.11), and $x_{2}+\frac{3}{2} y_{2}=\frac{4}{3} x_{3}+2 y_{3}+\frac{1}{4 b}$, then $v$ defined by (3.6) satisfies (3.8).

Note that the equations of Proposition 3.5 are not enough to characterize the vector $z_{2}$; the only equation relating $x_{2}$ and $y_{2}$ is $v(1,1,0)=-v(0,1,1)=\frac{1}{2} x_{2}+\frac{3}{4} y_{2}$. We then only have to prove that there exists a bounded solution $\left(z_{N}\right)_{N \geq 3}$ to the system of equations (3.10) and (3.11). Note that if $z_{3}$ is fixed then, for all $N, z_{N}$ is fixed, recursively. Finding a bounded solution of this system of equations is then equivalent to finding an initial condition $z$ (necessarily unique by Proposition 3.4) such that if $z_{3}=z$ then $\left(z_{N}\right)_{N \geq 3}$ is bounded. 
4.1.1. The one-order recurrence relationship satisfied by $\left(z_{N}\right)_{N}$. We change the two-order recurrence system of (3.10) and (3.11) into a one-order recurrence relationship, so that we can easily express $z_{N}$ as a function of $z_{3}$, and vice versa. We easily find that $z_{N}$ satisfies the recurrence relationship

$$
B_{N} z_{N+1}=\left(C_{N}+K_{N}\right) z_{N}+\sum_{k=3}^{N}(-1)^{N-k} E(k, N) f_{k} \quad \text { for all } N \geq 3 .
$$

More precisely, (4.1) is satisfied for $N=3$ if $K_{3}=\tilde{C}_{3}-C_{3}$ and $E(3,3)=I_{2}$. Moreover, if it is true for a given $N \geq 3$ then it is true for $N+1$ as long as $K_{N+1}=D_{N+1}\left(C_{N}+K_{N}\right)^{-1} B_{N}$, $E(N+1, N+1)=I_{2}$, and $E(k, N+1)=D_{N+1}\left(C_{N}+K_{N}\right)^{-1} E(k, N)$ for all $k \in[3, N]$. Then recurrence relationship (4.1) is satisfied for every $N$ as soon as we can define two sequences of matrices $\left(K_{N}\right)_{N \geq 3}$ and $(E(k, N))_{N \geq 3,2 \leq k \leq N}$ such that

$$
\begin{gathered}
K_{N}=D_{N}\left(C_{N-1}+K_{N-1}\right)^{-1} B_{N-1} \quad \text { for all } N \geq 4, \\
K_{3}=\tilde{C}_{3}-C_{3}, \\
E(k, N)=D_{N}\left(C_{N-1}+K_{N-1}\right)^{-1} E(k, N-1) \quad \text { for all } k \in[3, N-1], \\
E(k, k)=I_{2} \quad \text { for all } k \geq 3 .
\end{gathered}
$$

We then have to recursively prove that $F_{N}:=K_{N}+C_{N}$ is invertible for all $N \geq 3$. We first prove this when $c$ is large enough compared to $b$.

4.1.2. Proof of the invertibility of $K_{N}+C_{N}$. Let us define

$$
V_{N}:=\left(\begin{array}{cc}
0 & \frac{1}{N} \\
1 & 0
\end{array}\right)
$$

Then $F_{N}=(b+d+c(N-1)) V_{N}+K_{N}$. We now define the matrix $G_{N}:=V_{N}+K_{N} / b$. Then

$$
F_{N}=(d+c(N-1)) V_{N}+b G_{N}=(d+c(N-1)) V_{N}\left(I_{2}+\frac{b}{d+c(N-1)} V_{N}^{-1} G_{N}\right) .
$$

Using the matricial norm $\|M\|=\sup _{i \in\{1,2\}}\left(\left|M_{i, 1}\right|+\left|M_{i, 2}\right|\right)$, note that $\left\|V_{N}^{-1}\right\|=N$.

Lemma 4.2. If $b \leq c / 24$, then $F_{N}$ is invertible and $\left\|G_{N}\right\| \leq 9$ for all $N \geq 4$.

This result will be generalized in Subsection 4.2 to all possible parameters $b, d$, and $c$.

Proof of Lemma 4.2. We prove it recursively. For $N=4$, we can compute the norm of $G_{4}$. Indeed, we have

$$
G_{4}=V_{4}+\frac{1}{b} D_{4} \tilde{C}_{4}^{-1} B_{3}
$$

which gives

$$
G_{4}=\left(\begin{array}{ll}
g_{11} & g_{12} \\
g_{21} & g_{22}
\end{array}\right)
$$


where

$$
\begin{aligned}
& g_{11}=-\frac{d+3 c}{48(b+d+2 c)}, \\
& g_{12}=\frac{1}{4}-\frac{9(d+3 c)}{64(b+d+2 c)}, \\
& g_{21}=1-\frac{d+3 c}{16(b+d+2 c)}-\frac{10(d+3 c)}{16(b+(d+2 c) / 3)}-\frac{(d+2 c)(d+3 c)}{8(b+d+2 c)(b+(d+2 c) / 3)}, \\
& g_{22}=-\frac{27}{64} \frac{(d+3 c)(b+2(d+2 c)+(d+2 c) / 3)}{(b+d+2 c)(b+(d+2 c) / 3)}+\frac{d+3 c}{32(b+(d+2 c) / 3)} .
\end{aligned}
$$

So

$$
\begin{aligned}
\left\|G_{4}\right\| & \leq \sup \left\{\frac{d+3 c}{d+2 c}\left(\frac{1}{48}+\frac{1}{4}+\frac{9}{64}\right), \frac{d+3 c}{d+2 c}\left(1+\frac{1}{16}+\frac{30}{16}+\frac{3}{8}+\frac{1}{64}\right)\right\} \\
& =\frac{d+3 c}{d+2 c} \frac{212}{64} \\
& \leq \frac{212}{64} \frac{3}{2} \\
& \leq 9 .
\end{aligned}
$$

For all $N$, the invertibility of the matrix $F_{N}$ is a consequence of $\left\|G_{N}\right\| \leq 9$. Indeed, if $\left\|G_{N}\right\| \leq 9$ then, as long as $b<c / 12$,

$$
\left\|\frac{b V_{N}^{-1} G_{N}}{d+c(N-1)}\right\| \leq \frac{9 b N}{d+c(N-1)}<1 .
$$

In this case, $I_{2}+b V_{N}^{-1} G_{N} /(d+c(N-1))$ is invertible, and so is $F_{N}$. Now let us assume that $\left\|G_{N}\right\| \leq 9$ for a given $N \geq 4$ and let us prove that $\left\|G_{N+1}\right\| \leq 9$. If $\left\|G_{N}\right\| \leq 9$ then $F_{N}$ is invertible and we can write $G_{N+1}=V_{N+1}+(1 / b) D_{N+1} F_{N}^{-1} \bar{B}_{N}$. Hence,

$$
G_{N+1}=V_{N+1}+D_{N+1}\left(I_{2}+\frac{b V_{N}^{-1} G_{N}}{d+c(N-1)}\right)^{-1} \frac{V_{N}^{-1}}{d+c(N-1)} \frac{B_{N}}{b} \text {. }
$$

Moreover, as long as $b \leq c / 24$,

$$
\begin{aligned}
\left\|\left(I_{2}+\frac{b V_{N}^{-1} G_{N}}{d+c(N-1)}\right)^{-1}\right\| & \leq \frac{1}{1-\left\|b V_{N}^{-1} G_{N} /(d+c(N-1))\right\|} \\
& \leq \frac{1}{1-9 b N /(d+c(N-1))} \\
& \leq 2 .
\end{aligned}
$$

Finally, for all $N \geq 4,\left\|D_{N+1}\right\| \leq d+c N$ and $\left\|V_{N}^{-1} B_{N}\right\| \leq 3 b$, which implies that

$$
\left\|G_{N+1}\right\| \leq 1+6\left(1+\frac{c}{d+3 c}\right) \leq 9 .
$$

This completes the proof.

As long as $b \leq c / 24,(4.1)$ is satisfied, which allows us to easily express $z_{N}$ as a function of $z_{3}$ for all $N \geq 3$. We now prove that there exists a real number $z$ such that if $z_{3}=z$ then $\left(z_{N}\right)$ is bounded. 
4.1.3. Boundedness of $z$. Let us assume here that $b<c / 24$, so that we can use the previous results. Setting

$$
M_{N}:=B_{N}^{-1}\left(C_{N}+K_{N}\right) \quad \text { and } \quad g_{N}:=\sum_{k=3}^{N}(-1)^{N-k} B_{N}^{-1} E(k, N) f_{k},
$$

we obtain

$$
z_{N+1}=M_{N} M_{N-1} \cdots M_{3}\left(z_{3}+\sum_{l=3}^{N} M_{3}^{-1} \cdots M_{l}^{-1} g_{l}\right)=P_{N}\left(z_{3}+\sum_{l=3}^{N} P_{l}^{-1} g_{l}\right)
$$

if $P_{N}=M_{N} M_{N-1} \cdots M_{3}$. To obtain the behavior of $\left(z_{N}\right)$, we then study $P_{N}$ and $g_{N}$.

Lemma 4.3. It holds that $\left\|M_{N}^{-1}\right\| \leq 2 b / c N$ if $N$ is large enough.

Proof. We previously proved (Lemma 4.2) that, for all $N \geq 3,\left\|G_{N}\right\| \leq 9$, with $G_{N}=$ $V_{N}+K_{N} / b$. Then, for all $N \geq 3,\left\|K_{N}\right\| \leq 10 b$. So, if $b<c / 24$, we have

$$
\left\|K_{N}\right\|<\frac{1}{2} c
$$

for all $N \geq 3$. Besides, the equation $K_{N+1}=D_{N+1}\left(C_{N}+K_{N}\right)^{-1} B_{N}$ can be detailed, and using (4.3), we obtain (see Appendix B)

$$
K_{N+1}=-b\left(\begin{array}{cc}
\frac{1}{2 N^{2}}+O\left(\frac{1}{N^{3}}\right) & \frac{1}{N}+O\left(\frac{1}{N^{3}}\right) \\
1+O\left(\frac{1}{N^{2}}\right) & \frac{3}{N^{2}}+O\left(\frac{1}{N^{3}}\right)
\end{array}\right) .
$$

Next,

$$
D_{N+1}^{-1}=\frac{N^{2}}{(d+c N)(N-2)(N-1)}\left(\begin{array}{cc}
\frac{3}{N} & -\frac{N-2}{N} \\
-(N-1) & 0
\end{array}\right) .
$$

We deduce from this that

$$
M_{N}^{-1}=D_{N+1}^{-1} K_{N+1}=\frac{b}{c}\left(\begin{array}{cc}
\frac{1}{N}+O\left(\frac{1}{N^{2}}\right) & O\left(\frac{1}{N^{3}}\right) \\
\frac{1}{2 N^{2}}+O\left(\frac{1}{N^{3}}\right) & \frac{1}{N}+O\left(\frac{1}{N^{2}}\right)
\end{array}\right) .
$$

This completes the proof.

Note that, if $N$ is large enough,

$$
\left\|M_{N}^{-1} M_{N+1}^{-1}\right\| \leq \frac{4 b^{2}}{c^{2} N^{2}} .
$$

Besides, we have the following lemma for $\left(g_{N}\right)_{N}$.

Lemma 4.4. $g$ satisfies

$$
g_{N}=C+\frac{C^{\prime}}{N}+o\left(\frac{1}{N}\right)
$$


Proof. From $g_{N}:=\sum_{k=3}^{N}(-1)^{N-k} B_{N}^{-1} E(k, N) f_{k}$ we deduce that

$$
g_{N+1}=-B_{N+1}^{-1} K_{N+1} g_{N}+B_{N+1}^{-1} f_{N+1} \text {. }
$$

Moreover,

$$
B_{N+1}^{-1}=\frac{1}{b} \frac{2 N(N+2)^{2}}{3+\left(2 N^{2}+8 N+3\right)\left(2 N^{2}+4 N-1\right)}\left(\begin{array}{cc}
\frac{3}{N+2} & \frac{2 N^{2}+8 N+3}{N+2} \\
2 N^{2}+4 N-1 & -1
\end{array}\right)
$$

and (4.4) yields

$$
-B_{N}^{-1} K_{N}=\left(\begin{array}{cc}
1+O\left(\frac{1}{N^{2}}\right) & \frac{3}{N^{2}}+O\left(\frac{1}{N^{3}}\right) \\
O\left(\frac{1}{N^{2}}\right) & 1+O\left(\frac{1}{N^{2}}\right)
\end{array}\right) .
$$

Equations (4.8), (4.9), and (4.10) yield the result.

Finally, we consider $\sum P_{l}^{-1} g_{l}$. Let us recall that $P_{l}=M_{l} M_{l-1} \cdots M_{3}$. We have

$$
\sum_{l=3}^{N}\left\|P_{l}^{-1} g_{l}\right\| \leq \sum_{l=3}^{N}\left\|P_{l}^{-1}\right\|\left\|g_{l}\right\|
$$

From (4.7) and Lemma 4.3, $\left(g_{l}\right)_{l \geq 3}$ is bounded and there exists a constant $C_{2}$ such that $\left\|M_{N}^{-1}\right\| \leq$ $C_{2} / N$ when $N$ is large enough. Then $\sum_{l=3}^{N} P_{l}^{-1} g_{l}$ converges and we define its limit

$$
z=\sum_{l=3}^{\infty} P_{l}^{-1} g_{l}
$$

The quantity $z$ is the initial condition we need to obtain a bounded solution to (3.10) and (3.11).

Lemma 4.5. The sequence $\left(z_{N}\right)_{N \geq 3}$ satisfying (3.10) and(3.11), and such that $z_{3}=-z$ (where $z$ is as defined in (4.11)), is bounded.

Proof. From (4.2),

$$
\begin{aligned}
z_{N+1} & =-P_{N}\left(\sum_{l=N+1}^{\infty} P_{l}^{-1} g_{l}\right) \\
& =-\sum_{l=N+1}^{\infty} M_{N+1}^{-1} M_{N+2}^{-1} \cdots M_{l}^{-1} g_{l} \\
& =-M_{N+1}^{-1} g_{N+1}-M_{N+1}^{-1} M_{N+2}^{-1} \sum_{l=N+2}^{\infty}\left(M_{N+3}^{-1} \cdots M_{l-2}^{-1}\right)\left(M_{l-1}^{-1} M_{l}^{-1}\right) g_{l} .
\end{aligned}
$$

By Lemmas 4.4 and 4.3 and (4.6), if $N$ is large enough, there exists a constant $C$ independent from $b$ such that

as required.

$$
\left\|z_{N}\right\| \leq C \frac{2 b}{c N}
$$

This completes the proof of Proposition 3.5 for small $b$. In the next subsection we generalize this result to any $b$. 


\subsection{Generalization to all possible values of $\boldsymbol{b}$}

Theorem 4.1. For all $(k, m, n)$ such that $k+m+n \geq 2, v(k, m, n)$ is an analytic function of b on $\mathbb{R}^{+*}$.

Corollary 4.1. For all demographic parameters $b>0$, $d$, and $c>0$, Proposition 3.5 holds.

Proof. From the end of Section 4.1.3, there exists a constant $K>0$ such that, if $b<K c$, (3.6) holds, which gives

$$
\begin{aligned}
& y_{N}=\frac{N^{2}}{4(N-2)(N-1)} v(N-1,0,1), \\
& x_{N}=\frac{N}{N-1}\left[v(N-1,1,0)-\frac{2 N-1}{4(N-2)} v(N-1,0,1)\right] .
\end{aligned}
$$

As long as $b<K c$, we then have

$$
\begin{aligned}
v(k, m, n)= & \frac{m(k-n)}{N-1}\left[v(N-1,1,0)-\frac{2 N-1}{4(N-2)} v(N-1,0,1)\right] \\
& +(k-n) \frac{N^{2}-(k-n)^{2}}{4(N-2)(N-1)} v(N-1,0,1) .
\end{aligned}
$$

Now from Theorem 4.1, for all $(k, m, n)$ in $\mathbb{N}_{* *}^{3}, v(k, m, n)$ is an analytic function of $b$ on $\mathbb{R}^{+*}$. The equality of two analytic functions on $(0, K c)$, (4.15), extends on $\mathbb{R}^{+*}$. Then (3.9) holds for all $(k, m, n) \in \mathbb{N}_{* *}^{3}$ if $x_{N}$ and $y_{N}$ are defined for all $N \geq 3$ and all $b>0$, by (4.14). From Lemma 4.1, $\left(z_{N}\right)_{N \geq 3}$ satisfies (3.10) and (3.11), so Proposition 3.5 holds, completing the proof.

Before proving Theorem 4.1, we prove the following result.

Lemma 4.6. For every $(k, m, n)$ in $\mathbb{N}_{* *}^{3}$, there exists a strictly positive real number $\rho$ such that $\mathbb{E}_{k, m, n}\left((1+\rho)^{\mathcal{T}_{\Gamma}}\right)<\infty$.

Proof. We define the random number of returns of $\mathcal{Z}$ in $\{N=2\}$ before reaching $\Gamma$ by $L \in \mathbb{Z}_{+}$, and let $\mathcal{T}_{2}^{(i)}$ be the $i$ th time of return of $\mathcal{Z}$ in $\{N=2\}\left(T_{2}^{(0)}=0\right.$ and $\left.\mathcal{T}_{2}^{(1)}=\mathcal{T}_{\{2\}}\right)$. Then

$$
\begin{aligned}
& \mathbb{E}_{k, m, n}\left((1+\rho)^{\mathcal{T}_{\Gamma}}\right) \\
& \leq \sum_{l=0}^{\infty} \mathbb{E}_{k, m, n}\left((1+\rho)^{\mathcal{T}_{2}^{(l+1)}} \mathbf{1}_{\{L=l\}}\right) \quad \text { as } \mathcal{T}_{\Gamma} \mathbf{1}_{\{L=l\}} \leq \mathcal{T}_{2}^{(l+1)} \mathbf{1}_{\{L=l\}} \\
& \quad=\sum_{\substack { l=0 \\
\begin{subarray}{c}{\left(k^{\prime}, m^{\prime}, n^{\prime}\right) \notin \Gamma \mid k^{\prime}+m^{\prime}+n^{\prime}=2 \\
\text { or }\left(k^{\prime}, m^{\prime}, n^{\prime}\right)=(k, m, n){ l = 0 \\
\begin{subarray} { c } { ( k ^ { \prime } , m ^ { \prime } , n ^ { \prime } ) \notin \Gamma | k ^ { \prime } + m ^ { \prime } + n ^ { \prime } = 2 \\
\text { or } ( k ^ { \prime } , m ^ { \prime } , n ^ { \prime } ) = ( k , m , n ) } }\end{subarray}}^{\infty} \mathbb{E}_{k, m, n}\left((1+\rho)^{\mathcal{T}_{2}^{(l+1)}} \mathbf{1}_{Z_{\mathcal{T}_{2}^{(l)}}^{(l)}=\left(k^{\prime}, m^{\prime}, n^{\prime}\right)} \mathbf{1}_{\{L=l\}}\right) \\
& \leq \max _{\substack{\left(k^{\prime}, m^{\prime}, n^{\prime}\right) \notin \Gamma \mid k^{\prime}+m^{\prime}+n^{\prime}=2 \\
\text { or }\left(k^{\prime}, m^{\prime}, n^{\prime}\right)=(k, m, n)}} \mathbb{E}_{k^{\prime}, m^{\prime}, n^{\prime}}\left((1+\rho)^{\left.\mathcal{T}_{\{2\}}\right)} \sum_{l=0}^{\infty} \mathbb{E}_{k, m, n}\left((1+\rho)^{\mathcal{T}_{2}^{(l)}} \mathbf{1}_{\{L \geq l\}}\right),\right.
\end{aligned}
$$

by the strong Markov property in $\mathcal{T}_{2}^{(l)}$. We now define

$$
S=\max _{\substack{\left(k^{\prime}, m^{\prime}, n^{\prime}\right) \mid k^{\prime}+m^{\prime}+n^{\prime}=2 \\ \text { or }\left(k^{\prime}, m^{\prime}, n^{\prime}\right)=(k, m, n)}} \mathbb{E}_{k^{\prime}, m^{\prime}, n^{\prime}}\left((1+\rho)^{\mathcal{T}_{\{2\}}} \mathbf{1}_{\{L \geq 1\}}\right),
$$


and prove that, for every $l$,

$$
\mathbb{E}_{k, m, n}\left((1+\rho)^{\mathcal{T}_{2}^{(l)}} \mathbf{1}_{\{L \geq l\}}\right) \leq S^{l}
$$

The result obviously holds for $l=0$ and is proved to hold recursively for every $l$ by using the strong Markov property in $T_{2}^{(l-1)}$, as previously. Now, from Proposition 2.1, for every $(k, m, n)$, there exists $\rho>0$ such that $\mathbb{E}_{k, m, n}\left((1+\rho)^{\left.\mathcal{T}_{\{2\}}\right)<\infty}\right.$. Then, by the dominated convergence theorem, $\mathbb{E}_{k, m, n}\left((1+\rho)^{\mathcal{T}_{\{2\}}} \mathbf{1}_{\{L \geq 1\}}\right) \rightarrow \mathbb{P}_{k, m, n}(L \geq 1)<1$ as $\rho \rightarrow 0$. Hence, there exists $\rho_{0}$ such that if $\rho<\rho_{0}, S<1$ and then $\mathbb{E}_{k, m, n}\left((1+\rho)^{\mathcal{T}_{\Gamma}}\right)<\infty$.

Proof of Theorem 4.1. We need to study the dependence of the probability $u$ in the fecundity parameter $b$, so we denote by $u((k, m, n), \delta, b)$ the fixation probability of allele $a$ when $Z_{0}=$ $(k, m, n)$ and by $v((k, m, n), b)$ its derivative with respect to $\delta$. If $u((k, m, n), \cdot, \cdot)$ is an analytic function of $(b, \delta)$ on $\mathbb{R}^{+*} \times \mathbb{R}$, then $v((k, m, n), \cdot)$ is an analytic function of $b$ on $\mathbb{R}^{+*}$. Now

$$
u((k, m, n), \delta, b)=\sum_{l \geq 1} \sum_{\left(i_{1}, \ldots, i_{l}\right) \in S_{(k, m, n) \rightarrow \Gamma a}} \pi_{i_{1} i_{2}}^{\delta, b} \cdots \pi_{i_{l-1} i_{l}}^{\delta, b},
$$

where $\pi_{i_{k} i_{k+1}}^{\delta, b}$ is the transition probability from state $i_{k}$ to state $i_{k+1}$ and an analytic function of $(b, \delta)$ on $\mathbb{R}^{+*} \times \mathbb{R}$. Then $u$ is the simple limit of analytic functions on $\mathbb{R}^{+*} \times \mathbb{R}$. By Equations (9.13.1) and (9.13.2) of [9], a sequence of analytic functions $\left(f_{n}\right)_{n \in \mathbb{N}}$ defined on an open set $S$ of $\mathbb{C}$ which converges simply towards a function $f$ on $S$ is proved to converge uniformly on every compact subset of $S$ as long as $\left\{f_{n}, n \in \mathbb{N}\right\}$ is relatively compact. We extend the functions $\pi_{i_{k} i_{k+1}}^{\delta, b}$ on the open set $E_{1}^{\beta} \times E_{2}^{\beta}$, where $\beta \in \mathbb{R}^{+*}$ and

$$
\begin{aligned}
& E_{1}^{\beta}=\{z \in \mathbb{C}|\operatorname{Re}(z)>0,| \operatorname{Im}(z) \mid<\beta \operatorname{Re}(z)\}, \\
& E_{2}^{\beta}=\left\{z \in \mathbb{C}|\operatorname{Re}(z)|<\frac{1}{2} d,|\operatorname{Im}(z)|<\beta(d-|\operatorname{Re}(z)|+2 c)\right\} .
\end{aligned}
$$

We set $b=b_{\mathrm{r}}+\mathrm{i} b_{\mathrm{i}} \in E_{1}^{\beta}$ and $\delta=\delta_{\mathrm{r}}+\mathrm{i} \delta_{\mathrm{i}} \in E_{2}^{\beta}$, and denote by $P_{(k, m, n)\left(k^{\prime}, m^{\prime}, n^{\prime}\right)}^{b, \delta}$ the analytic extension of $\pi_{(k, m, n)\left(k^{\prime}, m^{\prime}, n^{\prime}\right)}^{b, \delta}$ on $E_{1}^{\beta} \times E_{2}^{\beta}$. For all $(b, \delta) \in E_{1}^{\beta} \times E_{2}^{\beta}$, and all $(k, m, n)$ and $\left(k^{\prime}, m^{\prime}, n^{\prime}\right)$ neighbors in $\mathbb{N}_{* *}^{3}$,

$$
\left|P_{(k, m, n)\left(k^{\prime}, m^{\prime}, n^{\prime}\right)}^{b, \delta}\right| \leq \sqrt{1+\beta^{2}} P_{(k, m, n)\left(k^{\prime}, m^{\prime}, n^{\prime}\right)}^{b_{r}, \delta_{r}}=\sqrt{1+\beta^{2}} \pi_{(k, m, n)\left(k^{\prime}, m^{\prime}, n^{\prime}\right)}^{b_{r}, \delta_{r}} .
$$

Indeed, let us make the computation if $\left(k^{\prime}, m^{\prime}, n^{\prime}\right)=(k, m-1, n)$ :

$$
\begin{aligned}
\left|P_{(k, m, n)(k, m-1, n)}^{b, \delta}\right| & =\left|\frac{(d+\delta+c(N-1)) m}{b N+d N+\delta m+c N(N-1)}\right| \\
& \leq \frac{|(d+\delta+c(N-1)) m|}{\operatorname{Re}(b N+d N+\delta m+c N(N-1))} \\
& =\frac{\sqrt{\left(d+\delta_{r}+c(N-1)\right)^{2} m^{2}+\delta_{i}^{2} m^{2}}}{b_{r} N+d N+\delta_{r} m+c N(N-1)} \\
& \leq \frac{\left(d+\delta_{r}+c(N-1)\right) m\left(\sqrt{1+\delta_{i}^{2} /\left(d+\delta_{r}+c(N-1)\right)^{2}}\right)}{b_{r} N+d N+\delta_{r} m+c N(N-1)}
\end{aligned}
$$




$$
\begin{aligned}
& \leq \frac{\left(d+\delta_{r}+c(N-1)\right) m \sqrt{1+\beta^{2}}}{b_{r} N+d N+\delta_{r} m+c N(N-1)} \quad\left(\text { since } \delta \in E_{2}^{\beta}\right) \\
& =\sqrt{1+\beta^{2}} P_{(k, m, n)(k, m-1, n)}^{b_{r}, \delta_{r}} .
\end{aligned}
$$

Similar computations are possible for other transitions. Then, as $\sqrt{1+\beta^{2}} \leq 1+\beta^{2}$,

$$
\begin{aligned}
\sum_{l \geq 1}^{L} \sum_{\left(i_{1}, \ldots, i_{l}\right) \in S_{(k, m, n) \rightarrow \Gamma_{a}}}\left|P_{i_{1} i_{2}}^{\delta, b} \cdots P_{i_{l-1} i_{l}}^{\delta, b}\right| & \leq \sum_{l \geq 1}^{L}\left(1+\beta^{2}\right)^{l} \sum_{\left(i_{1}, \ldots, i_{l}\right) \in S_{(k, m, n) \rightarrow \Gamma}} \pi_{i_{1} i_{2}}^{\delta_{r}, b_{r}} \cdots \pi_{i_{l-1} i_{l}}^{\delta_{r}, b_{r}} \\
& \leq \sum_{l \geq 1}^{L}\left(1+\beta^{2}\right)^{l} \mathbb{P}_{k, m, n}\left(\mathcal{T}_{\Gamma_{a}}=l\right) \\
& \leq \mathbb{E}_{k, m, n}\left(\left(1+\beta^{2}\right)^{\mathcal{T}_{\Gamma_{a}}} \mathbf{1}_{\left\{\mathcal{T}_{\Gamma_{a}}<\infty\right\}}\right) \\
& \leq \mathbb{E}_{k, m, n}\left(\left(1+\beta^{2}\right)^{\mathcal{T}_{\Gamma}}\right)
\end{aligned}
$$

since, if $\mathcal{T}_{\Gamma_{a}}<\infty, \mathcal{T}_{\Gamma_{a}}=\mathcal{T}_{\Gamma}$.

In the following subsection, we establish some properties of the derivative $v(k, m, n)$.

\subsection{Boundedness and the sign of $v$}

Proposition 4.1. (i) For all demographic parameters $b, d$, and $c$, $v$ is a bounded function of $(k, m, n)$.

(ii) Assume that $k \geq n$. Then $v_{k, m, n}=\mathbb{E}_{(k, m, n)}\left[\int_{0}^{T} L v\left(Z_{t}\right) \mathrm{d} t\right] \geq 0$, where $T=\inf \left\{t, k_{t}=\right.$ $n_{t}$ or $\left.m_{t}=n_{t}=0\right\}$.

(iii) $v(k, m, n)$ has the same sign as $k-n$.

Proof. Part (i) is a consequence of (4.13), and part (iii) is a consequence of part (ii). For (ii), by Proposition 3.5, it suffices to prove the result when $k>n$. With the function $v$ bounded in $(k, m, n)$ (by (i)), Dynkin's formula stopped at the stopping time $T$ gives

$$
\mathbb{E}_{k, m, n}\left[v\left(Z_{T}\right)\right]=v(k, m, n)-\mathbb{E}_{(k, m, n)}\left[\int_{0}^{T} L v\left(Z_{t}\right) \mathrm{d} t\right] .
$$

Using the fact that $v\left(Z_{T}\right)=0$ (from Proposition 3.5) yields the result.

Note that the sign of $\delta$ is not sufficient to determine whether allele $a$ has a larger fixation probability than a neutral allele. This property depends on the initial genetic repartition of the population: if there are more alleles $A$ (respectively $a$ ) initially then allele $a$ has a lower fixation probability than a neutral allele if and only if $\delta>0$ (respectively $\delta<0$ ). In Section 5 we will consider the particular case in which allele $a$ appears as a mutant in the population. In this case, at the mutation time, there is only one individual with genotype $A a$ and no individual with genotype $a a$, and so the population starts from a state of the form $(k, 1,0)$. As the derivative of $u$ with respect to $\delta$ is $-v$, the fixation probability of allele $a$ is

$$
u((k, 1,0), \delta)=\frac{1}{2(k+1)}-\delta\left(\frac{k}{k+1} x_{k+1}+\frac{k(2 k+1)}{(k+1)^{2}} y_{k+1}\right)+o(\delta) .
$$




\subsection{Proof of Proposition 3.6}

As in the computations for $v$, Proposition 3.6 holds if we can find a bounded sequence $\left(z_{N}^{\prime}\right)_{N \geq 2}$ which is the solution of (3.14) and (3.15). To prove this, we use a similar proof as for $\delta^{\prime}=0$ (Section 4.1). Setting

$$
h_{k}=f_{k}^{\prime} \quad \text { for all } k \geq 4, \quad h_{3}=f_{3}^{\prime}-D_{3}^{\prime} \tilde{C}_{2}^{\prime-1} \tilde{f}_{2}^{\prime},
$$

we easily obtain, for all $N \geq 3$,

$$
B_{N}^{\prime} z_{N+1}^{\prime}=\left(C_{N}^{\prime}+K_{N}^{\prime}\right) z_{N}^{\prime}+\sum_{k=3}^{N}(-1)^{k} E^{\prime}(N, k) h_{k}
$$

with

$$
\begin{aligned}
K_{3}^{\prime} & =D_{3}^{\prime} \tilde{C}_{2}^{\prime-1} \tilde{B}_{2}^{\prime} \\
K_{N}^{\prime} & =D_{N}^{\prime}\left(C_{N-1}^{\prime}+K_{N-1}^{\prime}\right)^{-1} B_{N-1}^{\prime} \quad \text { for all } N \geq 4
\end{aligned}
$$

and

$$
\begin{aligned}
E^{\prime}(k, k) & =I_{2} \quad \text { for all } k \geq 3, \\
E^{\prime}(N, k) & =D_{N}^{\prime}\left(C_{N-1}^{\prime}+K_{N-1}^{\prime}\right)^{-1} E^{\prime}(N-1, k) \\
& =K_{N}^{\prime} B_{N-1}^{\prime-1} E^{\prime}(N-1, k) \quad \text { for all } N \geq k+1 .
\end{aligned}
$$

Note here that the detailed computation of $h_{3}$ shows that $h_{3}$ does not depend on $x_{2}$ and $y_{2}$ (which are not known) but only on $x_{2}+\frac{3}{2} y_{2}$. The only difficulty in adapting the proof of Section 4.1 is when proving that there exists a constant $C$ such that, for all $N,\left\|B_{N}^{\prime-1} h_{N}\right\| \leq C / N^{2}$. Note that we have

$$
\begin{aligned}
B_{N}^{\prime-1}= & \frac{N-1}{b} \frac{N+1}{\left(2 N^{2}-2 N-1\right)\left(N^{2}+N-3 / 2\right)+1 / 2} \\
& \times\left(\begin{array}{cc}
\frac{N^{2}+N-3 / 2}{N+1} & \frac{1}{N+1} \\
-\frac{1}{2} & 2 N^{2}-2 N-1
\end{array}\right) .
\end{aligned}
$$

From (4.5), (4.7), and (4.12),

$$
y_{N}=\frac{C_{1}}{N}+\frac{C_{2}}{N^{2}}+O\left(\frac{1}{N^{3}}\right) .
$$

Then

$$
\left\|\left(\begin{array}{cc}
\frac{N^{2}+N-3 / 2}{N+1} & \frac{1}{N+1} \\
-\frac{1}{2} & 2 N^{2}-2 N-1
\end{array}\right) h_{N}\right\|=O(1) \quad \text { and } \quad\left\|B_{N}^{\prime-1} h_{N}\right\|=O\left(\frac{1}{N^{2}}\right) .
$$

We now know that if the birth parameter $b$ is small enough compared to $c$, then $v^{\prime}$ is effectively defined as in (3.7). To generalize this result to all possible values of parameters $b$ and $c$, we adapt the proof of Theorem 4.1 and Corollary 4.1 to $\delta^{\prime}$, without any difficulty. Note here that, for all demographic parameters, $v^{\prime}$ is a positive bounded function of $(k, m, n)$. 


\subsection{Proof of the analyticity of $u(k, m, n)$}

To conclude these results, we now prove that $u\left((k, m, n), \delta, \delta^{\prime}\right)$ is an analytic function of $\left(\delta, \delta^{\prime}\right)$ in the neighborhood of $(0,0)$.

We use analytic extension arguments as in the proof of Theorem 4.1. Here $\delta$ and $\delta^{\prime}$ are complex numbers, denoted by $\delta=\delta_{\mathrm{r}}+\mathrm{i} \delta_{\mathrm{i}}$ and $\delta^{\prime}=\delta_{\mathrm{r}}^{\prime}+\mathrm{i} \delta_{\mathrm{i}}^{\prime}$. We take $\left(\delta, \delta^{\prime}\right) \in\left(E^{\beta}\right)^{2}$ with $E^{\beta}=\{z \in \mathbb{C}|| \operatorname{Re}(z)|<d / 2,| \operatorname{Im}(z) \mid<\beta(d-|\operatorname{Re}(z)|+2 c)\}$, and denote by $\pi_{(k, m, n)\left(k^{\prime}, m^{\prime}, n^{\prime}\right)}^{\delta,}$ the transition probability for $Z$ from $(k, m, n)$ to one of its neighbors $\left(k^{\prime}, m^{\prime}, n^{\prime}\right)$ and by $P_{(k, m, n)\left(k^{\prime}, m^{\prime}, n^{\prime}\right)}^{\delta, \delta^{\prime}}$ the analytic continuation of $\pi_{(k, m, n)\left(k^{\prime}, m^{\prime}, n^{\prime}\right)}^{\delta, \delta^{\prime}}$ on $\left(E^{\beta}\right)^{2}$. Then

$$
\left|P_{(k, m, n)\left(k^{\prime}, m^{\prime}, n^{\prime}\right)}^{\delta, \delta^{\prime}}\right| \leq\left(1+\beta^{2}\right) P_{(k, m, n)\left(k^{\prime}, m^{\prime}, n^{\prime}\right)}^{\delta_{r}, \delta_{r}^{\prime}}=\left(1+\beta^{2}\right) \pi_{(k, m, n)\left(k^{\prime}, m^{\prime}, n^{\prime}\right)}^{\delta_{r}, \delta_{r}^{\prime}},
$$

which completes the proof.

Theorem 3.1 is now proved.

\section{Mutational scale: convergence and extinction vortex}

Understanding and quantifying the extinction risk of a population is a very important issue, in particular within the framework of species conservation [19]. We now consider the phenomenona called 'mutational meltdown' [12]: within small populations, inbreeding favors the fixation of deleterious alleles that would disappear in an infinite size population [3], [8], [14]. This phenomenona is then characterized by more and more frequent fixations of deleterious alleles, which creates an extinction vortex and leads to a rapid extinction of the population [11], [19]. We wish now to observe this acceleration of mutation fixations. To this end, we introduce mutations in our model, and consider a different time scale.

\subsection{General model}

As introduced in Section 2, each individual is now characterized by its genotype $x \in \boldsymbol{G}:=$ $\left\{\{\mathcal{A}, \mathcal{C}, \mathcal{G}, \mathcal{T}\}^{G}\right\}^{2}$. Now every DNA strand can mutate during the individual lifetime, at rate $\mu_{K}:=\mu / K$. Here $K$ is a scaling parameter that will go to $\infty$, following a rare mutation hypothesis, which is usual in evolutionary genetics [1], [11]. For every $a, a^{\prime} \in\{\mathcal{A}, \mathcal{C}, \mathcal{g}, \mathcal{T}\}^{G}$, we denote by $M\left(a, a^{\prime}\right)$ the probability that a DNA strand $a$ mutates to $a^{\prime}$ knowing that $a$ mutates. The population can then be represented at time $t$ by

$$
Z^{K}: t \mapsto \sum_{i=1}^{N_{t}^{K}} \delta_{x_{t}^{i, K}}
$$

where $N_{t}^{K}$ is the size of population $Z^{K}$ at time $t$ and $x_{t}^{i, K}$ is the genotype of the $i$ th individual in population $Z^{K}$ at time $t ; Z_{t}^{K}$ belongs to the discrete space

$$
E=\left\{\sum_{i=1}^{N} \delta_{x_{i}}, N \in \mathbb{Z}_{+}, x_{i} \in \boldsymbol{G} \text { for all } i\right\},
$$

where $E$ is equipped with its discrete topology and the norm $r(\mu, v)=\sum_{x \in G}|\mu(x)-v(x)|$. We denote by $\mathbb{D}([0, \infty), E)$ the Skorokhod space of left-limited, right-continuous functions from $\mathbb{R}^{+}$to $E$, endowed with the Skorokhod topology. We denote by $b(x, Z)$ the birth rate of an individual with genotype $x$ in population $Z$, and assume that there exists a constant $\bar{C}$ such that, for every $Z$ with size $N, \sum_{x \in \boldsymbol{G}} b(x, Z) \leq \bar{C} N$. As in Section 2, individuals can die 
either naturally or due to competition with other individuals, and, when the population size reaches 2 , we assume that no death can occur. We denote by $d(x, Z)$ the death rate of a given individual with genotype $x$ in population $Z$, and assume that, for every $x, d(x, Z)$ is bounded below by some positive power of the population size. For all $K>0$ and all real bounded measurable functions $f$ on $E$, if $Z=\sum_{i=1}^{N} \delta_{x^{(i)}}$ with $x^{(i)}=\left(x_{1}^{(i)}, x_{2}^{(i)}\right)$, the generator of the Markov process $Z^{K}$ is

$$
\begin{aligned}
L^{K} f(Z)= & \sum_{x \in G} b(x, Z)\left(f\left(Z+\delta_{x}\right)-f(Z)\right) \\
& +\sum_{i=1}^{N} d\left(x_{i}, Z\right)\left(f\left(Z-\delta_{x^{(i)}}\right)-f(Z)\right) \\
& +\sum_{i=1}^{N} \frac{\mu}{K} \sum_{y \in\{\mathcal{A}, \mathcal{C}, \mathcal{G}, \mathcal{T}\}^{G}} M\left(x_{1}^{(i)}, y\right)\left(f\left(Z-\delta_{x^{(i)}}+\delta_{\left(y, x_{2}^{(i)}\right)}\right)-f(Z)\right) \\
& +\sum_{i=1}^{N} \frac{\mu}{K} \sum_{y \in\{\mathcal{A}, \mathcal{C}, \mathcal{G}, \mathcal{T}\}^{G}} M\left(x_{2}^{(i)}, y\right)\left(f\left(Z-\delta_{x^{(i)}}+\delta_{\left(x_{1}^{(i)}, y\right)}\right)-f(Z)\right) .
\end{aligned}
$$

Notation. When the population is monomorphic, i.e. every individual has the same genotype $x$, we assume that the population follows a neutral logistic birth-and-death process as presented in Section 3.2, and we respectively denote by $b(x), d(x)$, and $c(x)$ the birth, natural, and competition death rates (denoted by $b, d$, and $c$ in Section 3.2). For all demographic parameters $b, d$, and $c$, we also define the stationary law $l(\cdot, b, d, c)$ of the population size of this neutral logistic birth-and-death process. Here $l$ satisfies the stationary equations system:

$$
\begin{gathered}
b(N-1) l(N-1, b, d, c)+(d+c N)(N+1) l(N+1, b, d, c) \\
=N(b+d+c(N-1)) l(N, b, d, c) \text { for all } N \geq 3, \\
2 b l(2, b, d, c)=3(d+2 c) l(3, b, d, c) .
\end{gathered}
$$

Then, for all $N \geq 2$,

$$
l(N, b, d, c):=\frac{1}{N} \prod_{k=2}^{N-1} \frac{b}{d+k c} / \sum_{i=2}^{\infty} \frac{1}{i} \prod_{j=2}^{i-1} \frac{b}{d+j c} .
$$

We now rescale time as $K$ goes to $\infty$ in order to observe mutation apparitions. More precisely, with the mean time of apparition of a mutation being equal to $1 / \mu_{K} \sim K$, we accelerate time by multiplying $t$ by $K$.

\subsection{Convergence and the limiting process in the adaptive dynamics setting}

Theorem 5.1. For all $0<t_{1}<\cdots<t_{n}$, the $n$-tuple $\left(Z_{K t_{1}}^{K}, \ldots, Z_{K t_{n}}^{K}\right)$ converges in law towards the process $\left(N_{t_{1}} \delta_{S_{t_{1}}}, \ldots, N_{t_{n}} \delta_{S_{t_{n}}}\right)$.

(i) $\left(S_{t}\right)_{t>0}$ is a Markov jump process that jumps from a homozygous genotype $x^{(1)}=\left(x_{1}, x_{1}\right)$ to another homozygous genotype $x^{(2)}=\left(x_{2}, x_{2}\right)$, where $x_{1}$ and $x_{2}$ are in $\{\mathcal{A}, \mathcal{C}, \mathcal{g}, \mathcal{T}\}^{G}$, at rate $\tau\left(x^{(1)}, x^{(2)}\right)$. 
(ii) The rate

$$
\begin{aligned}
\tau\left(x^{(1)}, x^{(2)}\right)=2 \mu M\left(x_{1}, x_{2}\right) \sum_{N=2}^{\infty} & N f\left((N-1,1,0), x^{(1)}, x^{(2)}\right) \\
\times & l\left(N, b\left(x^{(1)}\right), d\left(x^{(1)}\right), c\left(x^{(1)}\right)\right),
\end{aligned}
$$

where $f\left((k, m, n), x^{(1)}, x^{(2)}\right)$ is the probability that, starting from $k$ individuals with genotype $x^{(1)}, m$ with genotype $\left(x_{1}, x_{2}\right)$, and $n$ with genotype $x^{(2)}$, the population finally becomes monomorphic with genotype $x^{(2)}$. In the particular case where only the natural death rate differs between individuals with genotypes $x^{(1)}$ and $x^{(2)}$, as in (3.3),

$$
f\left((N-1,1,0), x^{(1)}, x^{(2)}\right)=u\left((N-1,1,0), d\left(x_{1}, x_{2}\right)-d\left(x^{(1)}\right), d\left(x^{(2)}\right)-d\left(x^{(1)}\right)\right)
$$

with $d\left(x^{(1)}\right), d\left(\left(x_{1}, x_{2}\right)\right)$, and $d\left(x^{(2)}\right)$ the respective natural death rates of individuals with genotype $x^{(1)},\left(x_{1}, x_{2}\right)$, and $x^{(2)}$ (the generalization of genotypes $A A, A a$, and aa in Section 3.2), and $u$ as given in Section 3.

(iii) Conditionally to $\left(S_{t_{1}}, \ldots, S_{t_{n}}\right)=\left(x^{(1)}, \ldots, x^{(n)}\right)$, the random variables $N_{t_{1}}, \ldots, N_{t_{n}}$ are mutually independent and, for all $i, N_{t_{i}}$ has law $l\left(\cdot, b\left(x^{(i)}\right), d\left(x^{(i)}\right), c\left(x^{(i)}\right)\right)$.

At this mutational time scale, the process $\left(N_{t} \delta_{S_{t}}\right)_{t \geq 0}$ describes the successive fixations of mutations. Indeed, a jump of the limiting process $S$ corresponds to a change in the genotype of every individual of the population, i.e. a mutation fixation. Theorem 5.1 is proved similarly to the proof of Theorem 3.1 of [2]; see Appendix A.

\subsection{The extinction vortex}

In this section we focus on the jump process $S$ and assume that all mutations have the same effect as described in (3.3), i.e. when $x_{1}$ mutates to $x_{2}$, individuals with genotypes $x^{(1)},\left(x_{1}, x_{2}\right)$, and $x^{(2)}$ all have the same fecundity $b$ and competition parameter $c$, but

$$
d\left(x_{1}, x_{2}\right)=d\left(x^{(1)}\right)+\delta \quad \text { and } \quad d\left(x^{(2)}\right)=d\left(x^{(1)}\right)+\delta^{\prime} .
$$

What is more, we exclude overdominance cases by assuming that $\delta<\delta^{\prime}$. We denote by

$$
\tau\left(d, \delta, \delta^{\prime}\right)=\sum_{N=2}^{\infty} N u\left((N-1,1,0), d, \delta, \delta^{\prime}\right) l(N, d)
$$

the jump rate of the limiting process $S$ of Theorem 5.1 (5.2) when individuals have birth rate $b$, natural death rate $d$, and competition rate $c$ (the dependence on the parameters $b$ and $c$ is hidden, and, to simplify the notation, we assumed that $\mu=\frac{1}{2}$ ). This rate is also the rate of fixation of a deleterious mutation with size $\left(\delta, \delta^{\prime}\right)$. Let us recall that the extinction vortex is due to more and more rapid fixations of deleterious mutations in the population. We want to prove that the mean time to fixation of a deleterious mutation decreases when the number of already fixed mutations increases. When a deleterious mutation becomes fixed, the natural death rate of all individuals is increased by $\delta^{\prime}$. The vortex is then due to the fact that the mean time to fixation of a deleterious mutation is a decreasing function of the natural death rate $d$ of individuals, which we prove in the next theorem.

Theorem 5.2. Let us fix the demographic parameters $b$ and $c$ and assume that $\mathbb{E}\left(N_{0}^{2}\right)<$ $\infty$. If $\delta>0$ and $\delta^{\prime}>\delta$, and if $b$ is small enough, the mean time to a jump of process $S$, $T\left(b, d, c, \delta, \delta^{\prime}\right)=1 / \tau\left(b, d, c, \delta, \delta^{\prime}\right)$, is a decreasing function of $d$, the natural death rate of individuals. 
Here we underline the dependence of all quantities on $d$, by respectively denoting by $u\left((k, m, n), d, \delta, \delta^{\prime}\right), v((k, m, n), d)$, and $v^{\prime}((k, m, n), d)$ the fixation probability defined in Section 3 and its derivatives, when individuals have natural death rate $d$. We also denote by $l(\cdot, d)$ the stationary law of the population size (5.1). Before proving the theorem, we first prove the following lemma.

Lemma 5.1. If $d$ and $d^{\prime}$ are two nonnegative real numbers such that $d^{\prime}>d$, then there exists an integer $N_{0}$ such that, for all $N \leq N_{0}, l\left(N, d^{\prime}\right) \geq l(N, d)$, and, for all $N>N_{0}$, $l\left(N, d^{\prime}\right)<l(N, d)$.

Proof. Let $q(N)=l\left(N, d^{\prime}\right) / l(N, d)$. Equation (5.1) gives $q(N+1)=(d+c N) q(N) \times$ $\left(d^{\prime}+c N\right)^{-1}$. Then, if $d^{\prime}>d, q(N)$ is a strictly decreasing function of $N$. Next,

$$
q(2)=\frac{1}{2} \sum_{i=2}^{\infty} \frac{1}{i} \prod_{j=2}^{\infty} \frac{b}{d+j c} / \frac{1}{2} \sum_{i=2}^{\infty} \frac{1}{i} \prod_{j=2}^{\infty} \frac{b}{d^{\prime}+j c} ;
$$

hence, $q(2)>1$. Finally, if $q(N)>1$ for all $N$ then $l\left(N, d^{\prime}\right)>l(N, d)$ for all $N$, which is absurd as $l(\cdot, d)$ and $l\left(\cdot, d^{\prime}\right)$ are probability measures. Then there exists an integer $N_{0}$ such that, for all $N>N_{0}, q(N)<1$ and, for all $N \leq N_{0}, q(N) \geq 1$.

Proof of Theorem 5.2. From Theorem 3.1, the mean time to fixation of a mutation is $T(d, \delta$, $\left.\delta^{\prime}\right)=1 / \tau\left(d, \delta, \delta^{\prime}\right)$ with

$$
\begin{aligned}
\tau\left(d, \delta, \delta^{\prime}\right)= & \frac{1}{2}-\left[\sum_{N=2}^{\infty} N\left(\delta v((N-1,1,0), d)+\delta^{\prime} v^{\prime}((N-1,1,0), d)\right) l(N, d)\right] \\
& +o\left(|\delta|+\left|\delta^{\prime}\right|\right),
\end{aligned}
$$

where the differentiability of the infinite sum in (5.3) is obtained as in the proof of Proposition 3.4. Then, if $d^{\prime}>d$,

$$
\begin{aligned}
\tau\left(d^{\prime}, \delta, \delta^{\prime}\right)-\tau\left(d, \delta, \delta^{\prime}\right)= & \sum_{N=2}^{\infty} N\left(\delta v((N-1,1,0), d)+\delta^{\prime} v^{\prime}((N-1,1,0), d)\right) l(N, d) \\
& -\sum_{N=2}^{\infty} N\left(\delta v\left((N-1,1,0), d^{\prime}\right)+\delta^{\prime} v^{\prime}\left((N-1,1,0), d^{\prime}\right)\right) l\left(N, d^{\prime}\right) \\
& +o\left(|\delta|+\left|\delta^{\prime}\right|\right) \\
= & \delta \sum_{N=2}^{\infty} N l(N, d)\left(v((N-1,1,0), d)-v\left((N-1,1,0), d^{\prime}\right)\right) \\
& -\delta \sum_{N=2}^{\infty} N v\left((N-1,1,0), d^{\prime}\right)\left(l\left(N, d^{\prime}\right)-l(N, d)\right) \\
& +\delta^{\prime} \sum_{N=2}^{\infty} N l(N, d)\left(v^{\prime}((N-1,1,0), d)-v^{\prime}\left((N-1,1,0), d^{\prime}\right)\right) \\
& -\delta^{\prime} \sum_{N=2}^{\infty} N v^{\prime}\left((N-1,1,0), d^{\prime}\right)\left(l\left(N, d^{\prime}\right)-l(N, d)\right) \\
& +o\left(|\delta|+\left|\delta^{\prime}\right|\right) .
\end{aligned}
$$


Defining $N_{0}$ as in Lemma 5.1, we obtain

$$
\begin{aligned}
\tau\left(d^{\prime}, \delta,\right. & \left.\delta^{\prime}\right)-\tau\left(d, \delta, \delta^{\prime}\right) \\
= & \delta \sum_{N=2}^{\infty} N l(N, d)\left(v((N-1,1,0), d)-v\left((N-1,1,0), d^{\prime}\right)\right) \\
& +\delta^{\prime} \sum_{N=2}^{\infty} N l(N, d)\left(v^{\prime}((N-1,1,0), d)-v^{\prime}\left((N-1,1,0), d^{\prime}\right)\right) \\
& -\delta \sum_{N=2}^{\infty}\left(N v\left((N-1,1,0), d^{\prime}\right)-N_{0} v\left(\left(N_{0}-1,1,0\right), d^{\prime}\right)\right)\left(l\left(N, d^{\prime}\right)-l(N, d)\right) \\
& -\delta^{\prime} \sum_{N=2}^{\infty}\left(N v^{\prime}\left((N-1,1,0), d^{\prime}\right)-N_{0} v^{\prime}\left(\left(N_{0}-1,1,0\right), d^{\prime}\right)\right)\left(l\left(N, d^{\prime}\right)-l(N, d)\right) \\
& +o\left(|\delta|+\left|\delta^{\prime}\right|\right) \quad(\text { the added terms being equal to } 0),
\end{aligned}
$$

which gives, if $w((k, m, n), d)=\delta v((k, m, n), d)+\delta^{\prime} v^{\prime}((k, m, n), d)$,

$$
\begin{aligned}
\tau\left(d^{\prime}, \delta,\right. & \left.\delta^{\prime}\right)-\tau\left(d, \delta, \delta^{\prime}\right) \\
= & \sum_{N=2}^{\infty} N l(N, d)\left(w((N-1,1,0), d)-w\left((N-1,1,0), d^{\prime}\right)\right) \\
& \quad-\sum_{N=2}^{\infty}\left(N w\left((N-1,1,0), d^{\prime}\right)-N_{0} w\left(\left(N_{0}-1,1,0\right), d^{\prime}\right)\right)\left(l\left(N, d^{\prime}\right)-l(N, d)\right) \\
& +o\left(|\delta|+\left|\delta^{\prime}\right|\right) .
\end{aligned}
$$

Let us now prove first that $N \mapsto N w\left((N-1,1,0), d^{\prime}\right)$ is increasing and then that $d \mapsto(w((N-$ $1,1,0), d)$ is decreasing. These two results imply Theorem 5.2 and will be consequences of the following two lemmas. We first prove, as in the proof of Theorem 4.1, that $u\left((k, m, n), \delta, \delta^{\prime}\right)$ is an analytic function of $\left(d, \delta, \delta^{\prime}\right)$ on $\mathbb{R}^{+} \times \mathbb{R}^{2}$; therefore, we can define the partial derivative $\partial w((k, m, n), d) / \partial d$ for all $(k, m, n) \in \mathbb{N}_{* *}^{3}$. Next, using Proposition 3.1, we prove, as in the proof of Proposition 3.4 and the proof of Theorem 4.1 of [2], that there exists a constant $C$ such that, for all $(k, m, n)$ such that $k+m+n=N$,

$$
\left|\frac{\partial w}{\partial d}(k, m, n)\right| \leq C N^{2} .
$$

Note that the infinitesimal generator $L$ (3.4) is the sum of two generators:

$$
(L f)(k, m, n)=\left(L_{b} f\right)(k, m, n)+\left(L_{d} f\right)(k, m, n) .
$$

Here

$$
\begin{aligned}
L_{b} f(Z)= & \sum_{i=1}^{3} b_{i}(Z)\left(f\left(Z+e_{i}\right)-f(Z)\right), \\
L_{d} f(Z)= & (d+c(N-1)) \\
& \times[k f(k-1, m, n)+m f(k, m-1, n)+n f(k, m, n-1)-N f(k, m, n)] .
\end{aligned}
$$


Since $\partial L w / \partial d=0$ (from (3.8) and (3.12)),

$$
\left(L \frac{\partial w(\cdot, d)}{\partial d}\right)(k, m, n)=\frac{-\left(L_{d} w(\cdot, d)\right)(k, m, n)}{d+c(N-1)} .
$$

Note also that

$$
\begin{aligned}
& \left(L_{d} w(\cdot, d)\right)(N-1,1,0) \\
& \quad=(d+c(N-1))[(N-1) w(N-2,1,0, d)-N w(N-1,1,0, d)],
\end{aligned}
$$

so if we prove that $\left(L_{d} w\left(\cdot, d^{\prime}\right)\right)(N-1,1,0) \leq 0$ for all $N \geq 2$ then $N \mapsto N w\left((N-1,1,0), d^{\prime}\right)$ is increasing. In fact, we prove the following result.

Lemma 5.2. If $b$ is small enough and $\delta^{\prime}>\delta$ then, for all $(k, m, n)$ in $\mathbb{N}_{* *}^{3}$,

$$
\left(L \frac{\partial w(\cdot, d)}{\partial d}\right)(k, m, n) \geq 0 .
$$

Proof. There exists a constant $C>0$ such that, for all $(k, m, n)$ in $\mathbb{N}_{* *}^{3}$,

$$
\begin{aligned}
(L w(\cdot, d))(k, m, n)= & \left(L_{d} w(\cdot, d)\right)(k, m, n)\left(1+\frac{b}{d+c(N-1)}\right) \\
& +\left(\left(L_{b} w(\cdot, d)\right)(k, m, n)-\frac{b}{d+c(N-1)}\left(L_{d} w(\cdot, d)\right)(k, m, n)\right) \\
= & -\frac{\delta m(k-n)+\delta^{\prime} n Y}{2 N(N-1)} \\
= & -\frac{\left(\delta^{\prime}-\delta\right) n m+k\left(\delta m+2 \delta^{\prime} n\right)}{2 N(N-1)} \\
\leq & \frac{-C(k m+m n+k n)}{2 N(N-1)} .
\end{aligned}
$$

Next, it follows from detailed computations that there exists a constant $C^{\prime}$ such that

$$
\begin{aligned}
& \left|\left(L_{b} w(\cdot, d)\right)(k, m, n)-\frac{b}{d+c(N-1)}\left(L_{d} w(\cdot, d)\right)(k, m, n)\right| \\
& \leq \delta b\left[|k-n|\left(m\left|x_{N+1}-x_{N-1}\right|+\frac{N^{2}-(k-n)^{2}}{N}\left|y_{N+1}-y_{N-1}\right|\right)\right] \\
& +\delta^{\prime} b\left[Y m\left|x_{N+1}-x_{N-1}\right|+m N\left|x_{N+1}^{\prime}-x_{N-1}^{\prime}\right|+(2 N-Y) Y\left|y_{N+1}-y_{N-1}\right|\right. \\
& \left.\left.\quad+(2 N-Y) Y\left|y_{N+1}^{\prime}-y_{N-1}^{\prime}\right|\right)\right] \\
& +b C^{\prime} \frac{k m+m n+k n}{N}\left(\left|x_{N+1}\right|+\left|x_{N-1}\right|+\left|x_{N+1}^{\prime}\right|+\left|x_{N-1}^{\prime}\right|\right. \\
& \left.\quad+\left|y_{N+1}\right|+\left|y_{N-1}\right|+\left|y_{N+1}^{\prime}\right|+\left|y_{N-1}^{\prime}\right|\right) .
\end{aligned}
$$

Now, from (4.5), (4.7), and (4.12), we obtain $\left\|z_{N+1}-z_{N-1}\right\| \leq C^{\prime \prime} / N^{2}$ for a constant $C^{\prime \prime}$. Finally from (4.12) and (4.13), when $b$ is small enough, there exists a constant $C^{\prime \prime \prime}$ independent from $b$ such that $\left\|z_{N+1}\right\|<C^{\prime \prime \prime} / N$. The same results are similarly proved for $Z^{\prime}$, then if $b$ is 
small enough,

$\left|L_{b} w(k, m, n)-\frac{b}{d+c(N-1)} L_{d} w(k, m, n)\right|<\frac{C(k m+m n+k n)}{2 N(N-1)} \quad$ for all $(k, m, n) \in \mathbb{N}_{* *}^{3}$, which gives $L_{d} w(k, m, n) \leq 0$ for all $(k, m, n)$ and the result by (5.7).

Lemma 5.3. If $b$ is small enough and $\delta^{\prime}>\delta$ then, for all $(k, m, n)$ in $\mathbb{N}_{* *}^{3}$,

$$
\frac{\partial w((k, m, n), d)}{\partial d}=-\mathbb{E}_{(k, m, n)} \int_{0}^{T_{\Gamma}}\left(L \frac{\partial w(\cdot, d)}{\partial d}\right)\left(Z_{t}\right) \mathrm{d} t .
$$

Proof. We use Dynkin's formula starting from $(k, m, n)=Z_{0}$ and stopped at time $T_{N}=$ $\inf \left\{t>0, N_{t} \geq N\right\}$ for $N \geq N_{0}$ :

$$
\mathbb{E}\left(\frac{\partial w\left(Z_{T_{\Gamma} \wedge T_{N}}, d\right)}{\partial d}\right)=\frac{\partial w\left(Z_{0}, d\right)}{\partial d}+\mathbb{E}\left(\int_{0}^{T_{\Gamma} \wedge T_{N}}\left(L \frac{\partial w(\cdot, d)}{\partial d}\right)\left(Z_{s}\right) \mathrm{d} s\right) .
$$

As $(L \partial w / \partial d(\cdot, d))(Z) \geq 0$ for all $Z \in \mathbb{N}_{* *}^{3}($ Lemma 5.2),

$$
\left(\int_{0}^{T_{\Gamma} \wedge T_{N}}\left(L \frac{\partial w(\cdot, d)}{\partial d}\right)\left(Z_{s}\right) \mathrm{d} s\right)_{N \geq N_{0}}
$$

is an increasing sequence of positive random variables since $T_{N} \leq T_{N+1}$ when $N \geq N_{0}=$ $k+m+n$. From the monotone convergence theorem, since $T_{\Gamma} \wedge T_{N} \rightarrow T_{\Gamma}$ as $N \rightarrow \infty$ almost surely (Proposition 2.2),

$$
\begin{aligned}
\mathbb{E}_{(k, m, n)} & {\left[\int_{0}^{T_{\Gamma} \wedge T_{N}}\left(L \frac{\partial w(\cdot, d)}{\partial d}\right)\left(Z_{s}\right) \mathrm{d} s\right] } \\
\rightarrow & \mathbb{E}_{(k, m, n)}\left[\int_{0}^{T_{\Gamma}}\left(L \frac{\partial w(\cdot, d)}{\partial d}\right)\left(Z_{s}\right) \mathrm{d} s\right] \text { as } N \rightarrow \infty
\end{aligned}
$$

Now, from (5.6), the process $\left(\partial w\left(Z_{t}, d\right) / \partial d\right)_{t \geq 0}$ is uniformly integrable as long as $\mathbb{E}\left(N_{0}^{2}\right)<$ $\infty$ from Proposition 2.2. As it is also a local submartingale, it converges in $L^{1}$ when $t$ goes to $+\infty$, which gives

$$
\mathbb{E}_{(k, m, n)}\left(\frac{\partial w\left(Z_{T_{\Gamma} \wedge T_{N}}, d\right)}{\partial d}\right) \rightarrow \mathbb{E}_{(k, m, n)}\left(\frac{\partial w\left(Z_{T_{\Gamma}}, d\right)}{\partial d}\right) \quad \text { as } N \rightarrow \infty .
$$

Using the fact that $\partial w\left(Z_{T_{\Gamma}}, d\right) / \partial d=0$ yields the result.

Finally, (5.7), (5.8), and Lemma 5.2 imply that $N \mapsto N w((N-1,1,0), d)$ is an increasing function of $N$, and it follows from Lemmas 5.2 and 5.3 that $w((N-1,1,0), d)$ is a decreasing function of $d$.

\subsection{Numerical results}

Equation (4.12) allows us to approximate the sequences $\left(z_{N}\right)_{N \geq 2}$ numerically, and we do the same for $\left(z_{N}^{\prime}\right)_{N \geq 2}$ and then for $\tau$ (5.4). In Figure 1 we plot the mean time $T$ to fixation of a deleterious mutation as a decreasing function of $d$ (Theorem 5.2) for various values of $b, \delta$, and $\delta^{\prime}$.

For more biological analysis and numerical results, we refer the reader to [7]. 


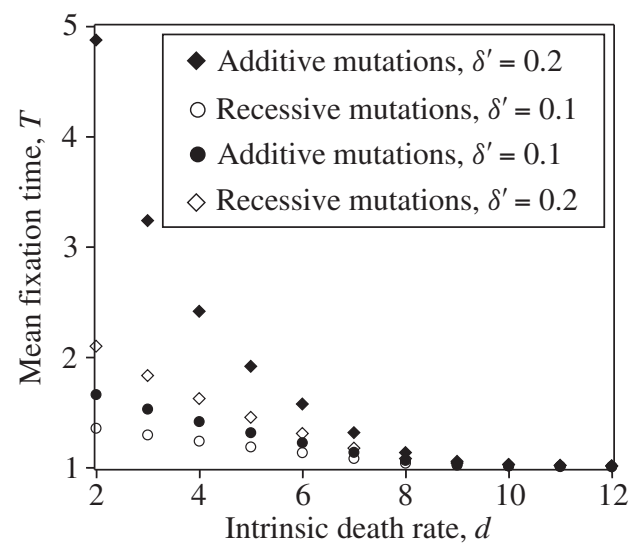

(a)

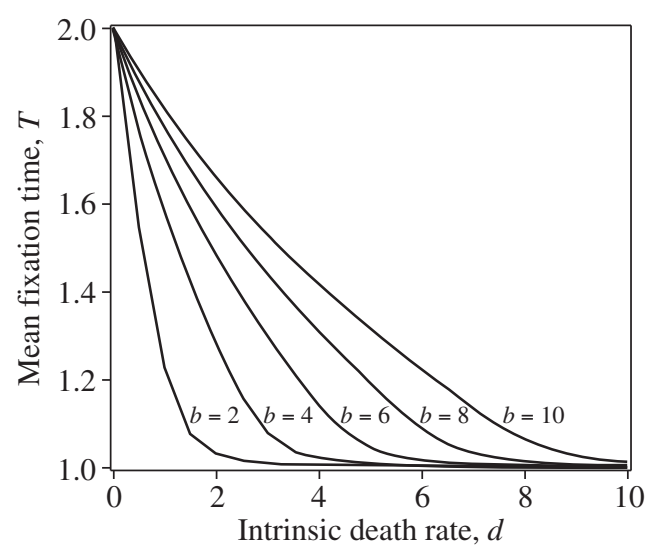

(b)

Figure 1: (a) The relationship between $T$, the mean time to fixation of a deleterious mutation, and the population intrinsic death rate $d$ as a function of selection and dominance: recessive mutations $(\delta=0)$ with $\delta^{\prime}=0.1$ (open circles) and $\delta^{\prime}=0.2$ (open diamonds), and additive mutations $\left(\delta=\delta^{\prime} / 2\right.$ ) with $\delta^{\prime}=0.1$ (filled circles) and $\delta^{\prime}=0.2$ (filled diamonds). Other demographic parameters are $b=10$, $c=0.1$, and $m=1$. (b) The relationship between the mean time to fixation of a deleterious mutation $T$ and the parameters $b$ and $d$. Each curve corresponds to a fixed value of $b$. Other parameters are $\delta=0.05$,

$$
\delta^{\prime}=0.1, c=0.1 \text {, and } m=1 .
$$

\section{Appendix A. Proof of Theorem 5.1}

In this paper we consider a diploid population and, as seen in Theorem 3.1, the diploidy generates interesting formulae for the fixation probability of a nonneutral allele. More precisely, this fixation probability is a function of the initial genetic repartition in the population (parameters $k, m$, and $n$ ) and cannot be reduced to a function of the initial numbers of alleles $A$ and $a$ in the population, as for a haploid population. At the mutational time scale (Section 5), this leads to mutation fixation rates that are different than those obtained in [2] for the haploid case.

The proof of Theorem 5.1 can however be seen as an extension of the proof of Theorem 3.1 of [2] to the cases where mutations occur during life and not at birth, and where no death can occur when there are two individuals in the population. We now explain why those differences do not hamper the proof of Theorem 3.1 of [2], which comprises three lemmas.

First lemma. Lemma 6.2 of [2] proves that there are no mutation accumulations when parameter $K$ goes to $\infty$. Using Proposition 2.2, the lemma and its proof also hold in our model.

Second lemma. The first part of Lemma 6.3 of [2] gives the limiting law of $K \tau_{1}$ and of the population size at time $\tau_{1}$ when $K$ goes to $\infty$, where $\tau_{1}$ is the first mutation apparition time for population $Z^{K}$. Here the proof is similar but uses different rates: as long as $t<\tau_{1}$, if the population is initially monomorphic with genotype $x$, the population size $\left(N_{t}^{K}\right)_{0<t<\tau_{1}}$ follows a birth-and-death process with birth rate $b\left(x, i \delta_{x}\right) i$ and death rate $d\left(x, i \delta_{x}\right) i$ when $N_{t}^{K}=i$, and $\tau_{1}$ is the first point of an inhomogeneous Poisson point process with intensity $(2 \mu / K) N_{t}^{K}$. Then, for any bounded function $f: \mathbb{N} \backslash\{1\} \rightarrow \mathbb{R}$,

$$
\begin{aligned}
\mathbb{E}\left(f\left(N_{\tau_{1}^{-}}^{K}\right) \mathbf{1}_{\left\{t \geq \tau_{1} / K\right\}}\right) & =2 \mu \int_{0}^{t} \mathbb{E}\left(f\left(N_{K s}^{K}\right) N_{K s}^{K} \exp \left(-2 \mu / K \int_{0}^{K s} N_{u}^{K} \mathrm{~d} u\right) \mathrm{d} s\right) \\
& =2 \mu \int_{0}^{t} \mathbb{E}\left(f\left(N_{K s}^{0}\right) N_{K s}^{0} \exp \left(-2 \mu / K \int_{0}^{K s} N_{u}^{0} d u\right) \mathrm{d} s\right),
\end{aligned}
$$


since the law of $N_{t}^{K}$ does not depend on $K$. The ergodic theorem finally gives

$$
\lim _{K \rightarrow \infty} \mathbb{E}^{K}\left(f\left(N_{\tau_{1}^{-}}^{K}\right) \mathbf{1}_{\left\{t \geq \tau_{1} / K\right\}}\right)=\frac{\mathbb{E}(N f(N))}{\mathbb{E}(N)} \int_{0}^{t} 2 \mu \mathbb{E}(N) \mathrm{e}^{-2 \mu \mathbb{E}(N) s} \mathrm{~d} s,
$$

where $N$ is a random variable with law $l$ defined by (5.1). The second part of Lemma 6.3 of [2] gives $\sup _{K>1} \mathbb{E}_{n \delta_{x}}^{K}\left(N_{\tau_{1}}^{p}\right)<\infty$. Here the proof needs to be slightly changed as the population size does not reach 1 in our model. We then define $L_{t}=\int_{0}^{t} \mathbf{1}_{\left\{N_{u}^{0}=2\right\}} \mathrm{d} u$ and have

$$
\mathbb{E}_{n \delta_{x}}^{K}\left(N_{\tau_{1}}^{p}\right) \leq 2 \mu \int_{0}^{\infty} \mathbb{E}\left(N_{K s}^{p+1} \exp \left(-\frac{2 \mu}{K} L_{K s}\right) \mathrm{d} s\right) .
$$

We finally prove that there exist $\lambda, \lambda^{\prime}, C>0$ such that $\mathbb{P}\left(L_{t} \leq \lambda t\right) \leq C \mathrm{e}^{-\lambda^{\prime} t}$ as in [2], by defining $s_{i}:=\inf \left\{s \geq t_{i-1}: N_{s}^{0}=2\right\}$ and $t_{i}=\inf \left\{t \geq s_{i}: N_{s}^{0}=3\right\}$.

Third lemma. The third lemma gives the behavior of $\rho_{1}$, the first time where the population becomes monomorphic, and $V_{1}$, the genotype of individuals at time $\rho_{1}$, if the population initially contains two genotypes $x$ and $y$. This lemma and the end of the proof of Theorem 5.1 are easily generalized to our model.

\section{Appendix B. Asymptotic behavior of $\left(K_{N}\right)_{N \geq 3}$}

The asymptotic behavior of $\left(K_{N}\right)_{N \geq 3}$ is obtained by several bootstrapping steps whose initial and central hypothesis is given by (4.3): $\left\|K_{N}\right\|<c / 2$ for all $N \geq 3$. Since $K_{N+1}=$ $D_{N+1}\left(C_{N}+K_{N}\right)^{-1} B_{N-1}$ for all $N \geq 4$, we obtain

$$
\begin{aligned}
K_{N+1}= & \frac{-b}{2(N+1)(N-1)} \frac{d+c N}{N} \\
& \times \frac{1}{\left((b+d+c(N-1)) / N+K_{12}^{N}\right)\left(b+d+c(N-1)+K_{21}^{N}\right)-K_{11}^{N} K_{22}^{N}} \\
& \times\left(\begin{array}{ll}
k_{11} & k_{12} \\
k_{21} & k_{22}
\end{array}\right),
\end{aligned}
$$

where

$$
\begin{aligned}
k_{11}= & \frac{N-2}{N}\left[b+d+c(N-1)+K_{21}^{N}-K_{11}^{N}\left(2 N^{2}-3\right)\right], \\
k_{12}= & \frac{N-2}{N}\left[\frac{\left.3 K_{11}^{N}+\left(b+d+c(N-1)+K_{21}^{N}\right)\left(2 N^{2}+4 N-3\right)\right]}{N+1}\right], \\
k_{21}= & (N-1)\left[\left(2 N^{2}-3\right)\left(\frac{b+d+c(N-1)}{N}+K_{12}^{N}\right)-K_{22}^{N}\right] \\
& +\frac{3}{N}\left[b+d+c(N-1)+K_{21}^{N}-K_{11}^{N}\left(2 N^{2}-3\right)\right], \\
k_{22}= & \frac{3}{N}\left[\frac{3 K_{11}^{N}+\left(b+d+c(N-1)+K_{21}^{N}\right)\left(2 N^{2}+4 N-3\right)}{N+1}\right] \\
& -\frac{N-1}{N+1}\left[K_{22}^{N}\left(2 N^{2}+4 N-3\right)+3\left(K_{12}^{N}+\frac{b+d+c(N-1)}{N}\right)\right] .
\end{aligned}
$$


Now, from (4.3), we know that $c+K_{12}^{N}$ does not get close to 0 when $N$ is large enough. We then easily obtain

$$
K_{N} \sim\left(\begin{array}{cc}
-\frac{\left(c-2 N K_{11}^{N}\right) b}{2 N^{2} c} & \frac{-b}{N} \\
-b & -\frac{\left(6 c-2 N K_{22}^{N}\right) b}{2 N^{2} c}
\end{array}\right) \quad \text { as } N \rightarrow \infty
$$

which gives

$$
K_{N}=\left(\begin{array}{cc}
o\left(\frac{1}{N}\right) & \frac{-b}{N}+o\left(\frac{1}{N}\right) \\
-b+o(1) & O\left(\frac{1}{N}\right)
\end{array}\right) \quad \text { as } N \rightarrow \infty,
$$

and then, from (B.1) again,

$$
K_{N}=\left(\begin{array}{cc}
o\left(\frac{1}{N^{2}}\right) & \frac{-b}{N}+o\left(\frac{1}{N}\right) \\
-b+o(1) & O\left(\frac{1}{N^{2}}\right)
\end{array}\right) \quad \text { as } N \rightarrow \infty .
$$

After some calculations, we finally obtain

$$
\begin{aligned}
& \frac{-b}{2(N+1)(N-1)} \frac{d+c N}{N} \\
& \times \frac{1}{\left((b+d+c(N-1)) / N+K_{12}^{N} t\right)\left(b+d+c(N-1)+K_{21}^{N}\right)-K_{11}^{N} K_{22}^{N}} \\
& \quad=-\frac{b}{2 c}\left[\frac{1}{N^{3}}+\frac{2 c-d}{c N^{4}}\right]+O\left(\frac{1}{N^{5}}\right)
\end{aligned}
$$

and

$$
\left(\begin{array}{ll}
k_{11} & k_{12} \\
k_{21} & k_{22}
\end{array}\right)=\left(\begin{array}{cc}
c N+O(1) & 2 c N^{2}+2 N(d-2 c)+O(1) \\
2 N^{3} c-2 N^{2}(2 c-d)+O(N) & 6 c N+O(1)
\end{array}\right),
$$

which gives the result.

\section{Acknowledgements}

I thank my $\mathrm{PhD}$ director Sylvie Méléard for her continual guidance during my work. I also would like to thank the anonymous reviewer for checking all the calculations and providing me with very constructive comments that helped to improve the manuscript. This work benefited from the support of the ANR MANEGE (ANR-09-BLAN-0215) and from the Chair 'Modélisation Mathématique et Biodiversité' of Veolia Environnement - École Polytechnique Museum National d'Histoire Naturelle - Fondation X.

\section{References}

[1] Champagnat, N. (2006). A microscopic interpretation for adaptive dynamics trait substitution sequence models. Stoch. Process. Appl. 116, 1127-1160.

[2] Champagnat, N. and Lambert, A. (2007). Evolution of discrete populations and the canonical diffusion of adaptive dynamics. Ann. Appl. Prob. 17, 102-155.

[3] Champagnat, N. And MÉlÉard, S. (2011). Polymorphic evolution sequence and evolutionary branching. Prob. Theory Relat. Fields 151, 45-94. 
[4] Champagnat, N., Ferrière, R. and Méléard, S. (2006). Unifying evolutionary dynamics: from individual stochastic processes to macroscopic models. Theoret. Pop. Biol. 69, 297-321.

[5] Collet, P., Martínez, S. and San Martín, J. (2013). Birth and death chains. In Quasi-Stationary Distributions. Springer, Berlin, pp. 66-111.

[6] Collet, P., Méléard, S. and Metz, J. A. J. (2013). A rigorous model study of the adaptive dynamics of Mendelian diploids. J. Math. Biol. 67, 569-607.

[7] Coron, C., Méléard, S., Porcher, E. And Robert, A. (2013). Quantifying the mutational meltdown in diploid populations. Amer. Naturalist 181, 623-636.

[8] Crow, J. And Kimura, M. (1970). An Introduction to Population Genetics Theory, 2nd edn. Harper and Row, New York.

[9] DieudonnÉ, J. (1969). Éléments d'Analyse, Vol. 1. Gauthier-Villars, Paris.

[10] Durrett, R. (2010). Probability Theory and Examples, 4th edn. Cambridge University Press.

[11] LANDE, R. (1994). Risk of population extinction from fixation of new deleterious mutations. Evolution 48, 1460-1469.

[12] LynCh, M., CONERy, J. AND Burger, R. (1995). Mutation accumulation and the extinction of small populations. Amer. Naturalist 146, 489-518.

[13] LynCH, M. ANd Gabriel, W. (1990). Mutation load and the survival of small populations. Evolution 44, 17251737.

[14] Metz, J. et al. (1996). Adaptive dynamics: a geometrical study of the consequences of nearly faithful reproduction. In Stochastic and Spatial Structures of Dynamical Systems, eds S. J. van Strien and S. M. VerduynLunel, North Holland, Amsterdam, pp. 183-231.

[15] Norris, J. (1997). Markov Chains. Cambridge University Press.

[16] ORR, H. A. (1998). The population genetics of adaptation: the distribution of factors fixed during adaptive evolution. Evolution 52, 935-949.

[17] ORR, H. A. (1999). The evolutionary genetics of adaptation: a simulation study. Genet. Res. 74, $207-214$.

[18] Seneta, E. And Vere-Jones, D. (1966). On quasi-stationary distributions in discrete-time Markov chains with a denumerable infinity of states. J. Appl. Prob. 3, 403-434.

[19] Soulé, M. E. (ed.) (1986). Conservation Biology: The Science of Scarcity and Diversity. Sinauer Associates, Sunderland, MA. 